\title{
A subset of SMN complex members have a specific role in tissue regeneration via ERBB pathway-mediated proliferation
}

\author{
Wuhong Pei ${ }^{1}$, Lisha $\mathrm{Xu}^{1}$, Zelin Chen ${ }^{1}$, Claire C. Slevin ${ }^{1}$, Kade P. Pettie $\mathbb{D}^{1}$, Stephen Wincovitch ${ }^{2}$, NISC Comparative Sequencing \\ Program* and Shawn M. Burgess (iD ${ }^{1 凶}$
}

\begin{abstract}
Spinal muscular atrophy (SMA) is the most common genetic disease in children. SMA is generally caused by mutations in the gene SMN1. The survival of motor neurons (SMN) complex consists of SMN1, Gemins (2-8), and Strap/Unrip. We previously demonstrated smn 1 and gemin 5 inhibited tissue regeneration in zebrafish. Here we investigated each individual SMN complex member and identified gemin3 as another regeneration-essential gene. These three genes are likely pan-regenerative, since they affect the regeneration of hair cells, liver, and caudal fin. RNA-Seq analysis reveals that smn 1, gemin3, and gemin 5 are linked to a common set of genetic pathways, including the tp53 and ErbB pathways. Additional studies indicated all three genes facilitate regeneration by inhibiting the ErbB pathway, thereby allowing cell proliferation in the injured neuromasts. This study provides a new understanding of the SMN complex and a potential etiology for SMA and potentially other rare unidentified genetic diseases with similar symptoms.
\end{abstract}

npj Regenerative Medicine (2020)5:6; https://doi.org/10.1038/s41536-020-0089-0

\section{INTRODUCTION}

Spinal muscular atrophy (SMA) is the leading hereditary cause of infant mortality ${ }^{1,2}$. The majority of SMA cases are caused by mutations in the survival of motor neuron 1 (SMN1) gene. SMN1 is ubiquitously expressed and a reduction of SMN1 protein leads to motor neuron death in patients afflicted with SMA. Although the incidence of SMA is 1:6000 in live births, the carrier frequency for a heterozygous SMN1 mutation can approach 1:40 in adults. Many important questions remain regarding the pathology of the disease, including why the ubiquitously expressed SMN1 protein primarily impacts motor neurons, which other organs are potentially affected by SMN1 deficiencies, and whether SMA is a developmental or degenerative disease (or both).

The SMN1 protein is part of the SMN complex, responsible for the assembly of small nuclear ribonucleoproteins (snRNP) needed for pre-mRNA splicing ${ }^{3,4}$. The SMN complex consists of nine proteins, however the majority of research on the complex has focused on the characterization of SMN1 and its role in SMA. In addition to its role in the SMN complex, SMN1 plays a role in many other biological processes, including axon growth, mRNA transport, ribosome biology, translational control, and maintaining intracellular homeostasis $^{5-8}$. Although there is some evidence showing that other SMN complex members, such as GEMIN3 and GEMIN5, also have functions independent of the SMN complex ${ }^{9-12}$, it remains largely unknown how the other SMN complex members relate to SMA, and whether other members have functions beyond the SMN complex. A reduction of the SMN1 protein in SMA results in the reduction of other SMN complex members ${ }^{13}$, suggesting that there is a functional inter-dependence among the nine genes.

In a previous study ${ }^{14}$, we showed that mutations in $s m n 1$ and gemin5 negatively impacted the ability of zebrafish to regenerate different tissues after injury. Regenerative medicine is a rapidly expanding field of science that focuses on replacing or regenerating organs damaged by injury, aging, or degenerative conditions. An active area of research within regenerative medicine is the restoration of hearing by replacing the lost mechanosensory receptors of the inner ear known as hair cells. Age-related hair cell death impairs the hearing of hundreds of millions of the elderly and hearing loss as a side-effect of therapeutic medications remains a major concern ${ }^{15}$. In general, mammals have very limited regenerative capability, however many non-mammal animal models including the zebrafish have been used extensively because they possess a much broader capacity for wound healing, including the capacity to regenerate hearing after damage. Zebrafish are particularly well suited for studying the regeneration of hair cells because of a second organ that fish and amphibians possess on their skin known as the lateral line, which consists of clusters of hair cells in structures known as "neuromasts" 16 . Similar to the case in the mammalian inner ear, hair cells in the neuromasts are surrounded by support cells, which in fish and amphibians can replace the lost hair cells through either mitotic division or trans-differentiation. Support cells in the zebrafish neuromast are further surrounded by mantle cells, which resemble quiescent stem or progenitor cells ${ }^{17}$. Hair cell regeneration studies in zebrafish have uncovered numerous genetic factors and molecular pathways that are associated with the regeneration of hair cells ${ }^{18}$; conversely, random mutagenesis studies revealed that mutations in only a small number of genes actually alter hair cell regeneration specifically ${ }^{14,17,19,20}$. There is consistently a gap between the number of genes transcriptionally associated with regeneration and the number of genes essential for regeneration in other tissues as well ${ }^{21,22}$. Therefore, seeking novel genes essential for tissue regeneration is pivotal in understanding the core molecular mechanisms of wound healing, and for ultimately advancing regenerative medicine.

In this study, we systematically mutated all nine genes encoding SMN complex proteins. Using hair cell regeneration in the zebrafish lateral line as an assay, we identified three SMN complex members as essential factors that regulate regeneration through ErbB pathway-mediated cell proliferation. Additional

\footnotetext{
Translational and Functional Genomics Branch, National Human Genome Research Institute, Bethesda, MD 20892, USA. ${ }^{2}$ Cytogenetics and Microscopy Core, National Human Genome Research Institute, Bethesda, MD 20892, USA. *A list of authors and their affiliations appears at the end of the paper. ${ }^{\circledR}$ email: burgess@mail.nih.gov
} 
studies revealed that these regenerative members were also essential for the regeneration of other tissues and all shared common transcriptional pathways altered in the mutant larvae. Our findings demonstrated a subset of the SMN complex proteins had separate functional roles involved in tissue regeneration.

\section{RESULTS}

Divergent roles for SMN complex members in embryo development and hair cell regeneration

Hearing loss is one of the common sensory disorders negatively affecting the quality of life for hundreds of millions of people worldwide $^{15}$. In a search for novel genes involved in hearing regeneration, we previously performed a large-scale mutagenesis screen in zebrafish and identified smn 1 and gemin 5 as essential genes for hair cell regeneration ${ }^{14}$. Both $\mathrm{Smn} 1$ and Gemin 5 belong to the SMN complex, a multiprotein complex functioning in the biosynthesis of snRNP. To investigate whether the regenerative abilities of Smn1 and Gemin5 are linked to the SMN complex activity, we independently mutated all nine genes in the SMN complex (Supplementary Table 1) and examined their involvement in hair cell regeneration. We found that in addition to smn1 and gemin5, mutations in gemin3 altered hair cell regeneration but had no effect on initial hair cell development (Fig. 1a, Supplementary Fig. 1a). We also found that mutations in the other six SMN complex genes, gemin2, gemin4, gemin6, gemin7, gemin8, and strap/unrip, had no impact on hair cell regeneration (Fig. 1d-i). None of the nine mutants showed an overt morphological phenotype in early larvae (data not shown), but all mutants except gemin8 and strap failed to survive to adulthood (Supplementary Table 2). Altogether, these data classified the functions of the nine members of the SMN complex into three categories: three genes (Smn1, Gemin3, and Gemin5) were essential for hair cell regeneration and adult survival, four genes (Gemin2, Gemin4, Gemin6, and Gemin7) were essential for adult survival but not for hair cell regeneration, and two genes (Gemin8 and Strap) neither impacted regeneration nor survival.

We generated an additional mutant allele for the three genes involved in regeneration, smn 1, gemin3, and gemin 5 to verify their roles in hair cell regeneration. The second alleles all recapitulated the deficits in hair cell regeneration (Fig. 1b, c, Supplementary Fig. $1 \mathrm{~b})$. To examine whether the regeneration was specific to the ablation of hair cells using $\mathrm{CuSO}_{4}$, we performed the ablation using neomycin and observed similar regeneration deficits (Supplementary Fig. 1c-e).

In support of their divergent phenotypes in hair cell regeneration and adult survival, whole-mount in situ hybridization analysis showed that the SMN complex genes possessed some common but also some different expression patterns at 3 days post fertilization (dpf) (Supplementary Fig. 2a-h). The brain expression of these genes in particular revealed both shared and specific expression patterns: five genes were restricted to a stripe at approximately the mid-hindbrain boundary. $s m n 1$ was enriched in the eye regions and gemin 5 was condensed at the midline of the brain; gemin3 expression was relatively weaker than the others; gemin7 and strap showed ubiquitous expression which was very different from the other gemins. At $1 \mathrm{dpf}$, a stronger similarity was detected in the brain expression between smn1 and gemin5 (Supplementary Fig. 2i). Both genes were enriched in the eyes, brain, and midline area. These differences in expression suggested that the gemins were not all necessarily expressed at stochiometric levels in all tissues.

Our whole-mount in situ hybridization analysis showed that none of the SMN complex genes were particularly enriched in the lateral line neuromasts (data not shown). However, single cell RNA-sequencing (RNA-Seq) analysis conducted by Lush et al. demonstrated that all these SMN complex genes are expressed at detectable levels in lateral line neuromasts, and different genes in the complex are expressed in different neuromast cell types at different levels (Supplementary Fig. 2j) ${ }^{17}$. The non-identical and complex patterns of gene expression for the different SMN subunits (as well as the different phenotypes) suggest that the various roles for each protein may not be simply as co-expressed subunits, but that the composition of the SMN complex and potentially alternate functions of the subunits may vary based on expression levels and cellular context.

Maternal mRNA and protein deposition allows zebrafish embryos to grow rapidly during the first few hours after birth and some maternal proteins can persist for days after fertilization. Although regeneration was analyzed at $7 \mathrm{dpf}$, we still examined whether the hair cell regeneration phenotype could be associated with the initial maternal deposition or a difference in the stability of mutant mRNAs. We analyzed the expression level of two regeneration genes (smn1 and gemin5) and three non-regeneration genes (gemin4, gemin6, and strap) at different stages of embryonic development by semiquantitative PCR and found no clear difference between these two groups of genes (Supplementary Fig. 2k), suggesting mRNA destabilization does not explain hair cell regeneration phenotypes or eventual larval death.

Genetic interactions have been observed among SMN complex genes ${ }^{23-25}$. To study whether there is a synergy among the three genes involved in regeneration, we generated an smn 1 and gemin5 double mutant and studied the effect of simultaneous depletion of two genes on morphology and hair cell regeneration. We found the smn1 and gemin5 double mutant had a normal embryonic morphology and normal hair cell development (data not shown), as observed in both the smn 1 and gemin5 single mutants. The double mutant showed the expected deficiency in hair cell regeneration; however, the level of deficiency was comparable to that of the gemin 5 mutant (Supplementary Fig. $3 a-c)$. Taken together, these data suggest there is no functional synergy among these regeneration genes, and smn 1 and gemin 5 appear to both be necessary and fall in the same regenerative pathway as the phenotypes in double mutants were neither synergistic nor additive.

Along with the SMN complex, the PRMT5 complex is also involved in the assembly of snRNP26,27. The PRMT5 complex comprises three members (PRMT5, MED50, and pICln) encoded by the prmt5, wdr77, and clns1a genes, respectively. To investigate whether the PRMT5 complex members are involved in regeneration, we analyzed neuromast hair cell regeneration in the embryos after knockdown of these genes by injecting Cas9 protein with targeting single guide RNAs (sgRNAs). gemin5 guide RNAs were used as a system control. Mutation frequency analysis showed that guide RNA injections led to efficient mutagenesis in all the targeted genes (Supplementary Fig. 3d). Reduced hair cell regeneration was detected in the embryos injected with gemin5 guide RNA, however, no alteration in hair cell regeneration was observed in the embryos injected with guide RNA targeting prmt5, wdr77, or clns1a (Supplementary Fig. 3e), suggesting the regeneration deficient phenotype observed in the mutants of the three SMN complex members is independent of snRNP assembly.

The overall neuromast size is smaller after hair cell ablation in mutants with regenerative phenotypes

We examined the neuromast cell patterning in the mutants and control siblings at 2 days post hair cell ablation to see if we could detect a difference in neuromast size in mutants using both transgenic labeling and immunohistochemical staining approaches. Double transgenic labeling of support cells by $\mathrm{Tg}$ (tnks 1 bp 1:EGFP) and hair cells by Tg(atoh1a:dTomato) in gemin5 mutants revealed that support cells in the mutant occupied a reduced area likely because of fewer cells and hair cells were fewer when compared to that of the control siblings (Fig. 2a). Whole 

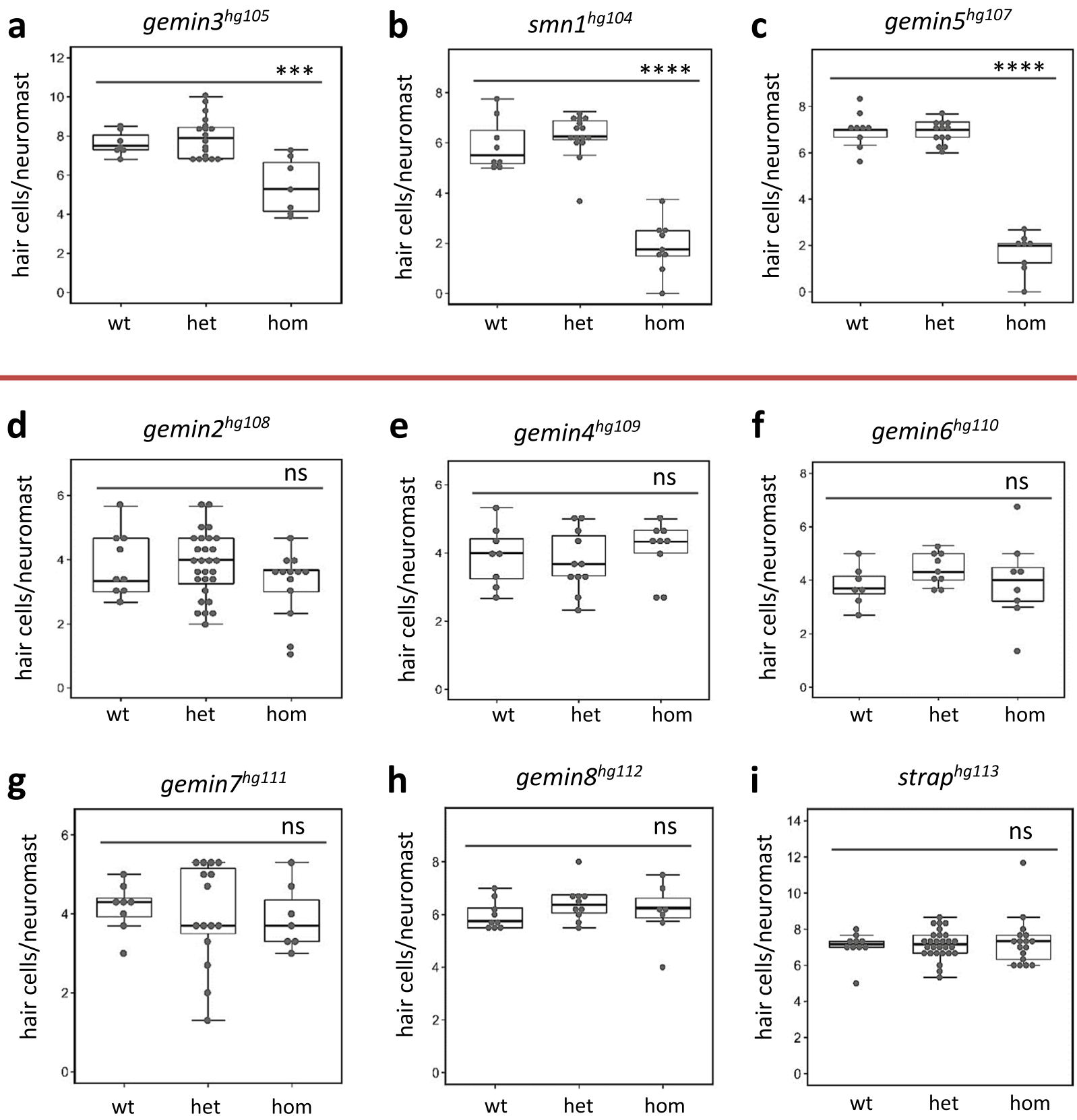

Fig. 1 Hair cell regeneration analysis for mutations in each gene of the SMN complex. Hair cell regeneration is impaired by homozygous

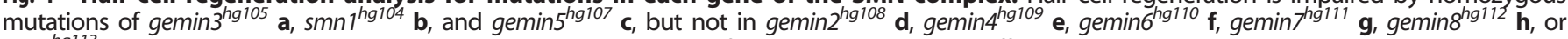
$\operatorname{strap}^{h 1113} \mathbf{i}$. Red line separates the mutations impacting regeneration from those that have no effect on regeneration. wt wild-type, het heterozygotes, hom homozygotes. Error bars in the graphs represent mean \pm s.e.m. The difference between wild-type and homozygote is labeled. ns, $P>0.05 ; * * *<<$ 0.001 ; ***P $P 0.0001$. Approximately 40 embryos were used for each of the regeneration analyses and then genotyped to study the genotype-phenotype correlation.

neuromast labeling using $\operatorname{Tg}(c / d n b$ :EGFP) showed that the size of the neuromast in the mutant was smaller than that of the control siblings (Fig. 2b). Alkaline phosphatase staining revealed that the structure of lateral line neuromasts were reduced in the mutant (Fig. 2c). Co-staining with anti-hair cell antibody and the nuclear stain DAPI revealed a reduction in the number of hair cells and neuromast cells (Fig. 2d, e).

We also used transgenes and immunohistochemical staining to examine the neuromast cells at 2 days post hair cell ablation in smn 1 and gemin3 mutants. Consistent with the results of the gemin 5 mutation, mutations in smn 1 and gemin 3 also caused a reduced area of support cells, impaired regeneration of hair cells and smaller neuromasts (Supplementary Fig. 4). All the data suggest the proliferative capacity in the neuromasts is reduced after injury preventing organ regrowth.

Regenerative-deficient mutants show reduced proliferation after injury

To directly test proliferative capacity of the support cells in the mutant neuromasts, we used an EdU incorporation assay to label the proliferation of neuromast cells after hair cell ablation. Compared to the control siblings, all three mutants possessed a reduced number of EdU-positive cells (Fig. 3), suggesting that after hair cell ablation, the mutants lack the capacity to effectively proliferate either their support cells or mantle cells. 
a
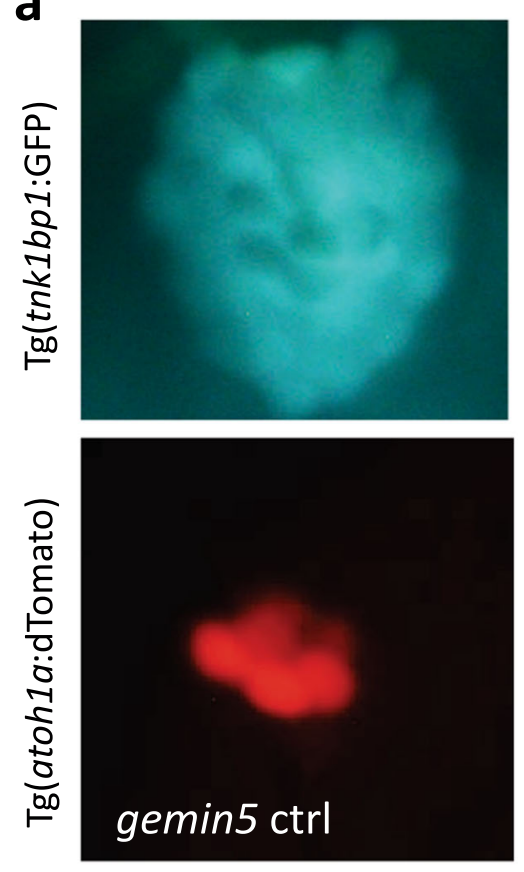
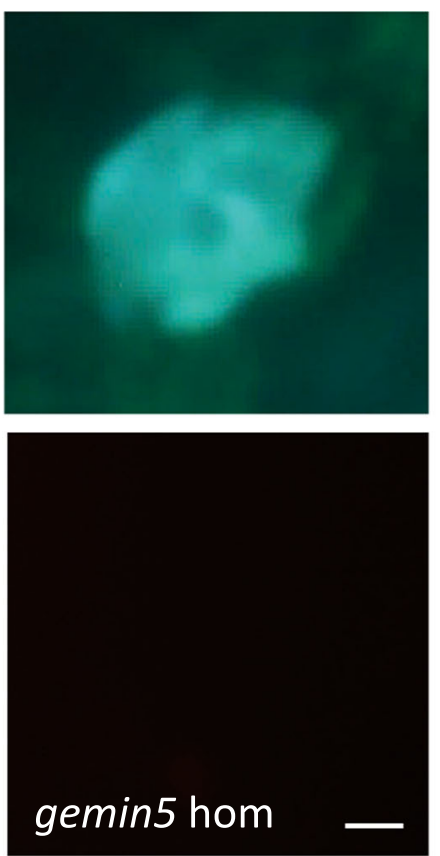

b
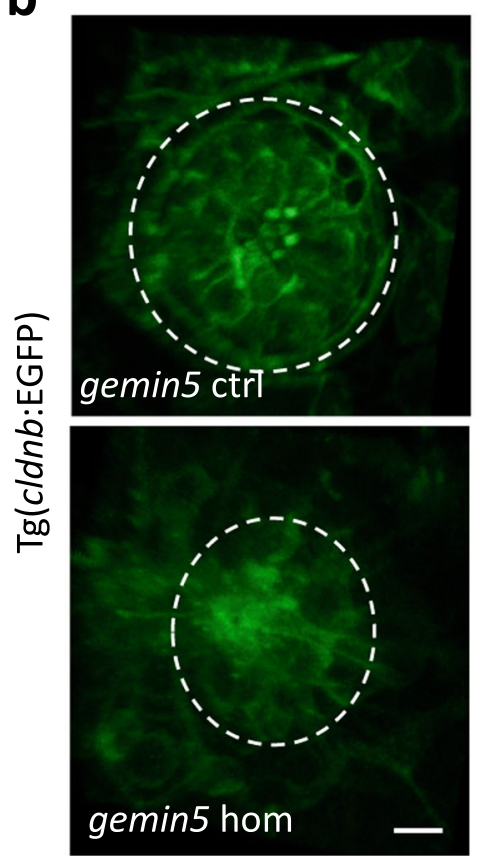
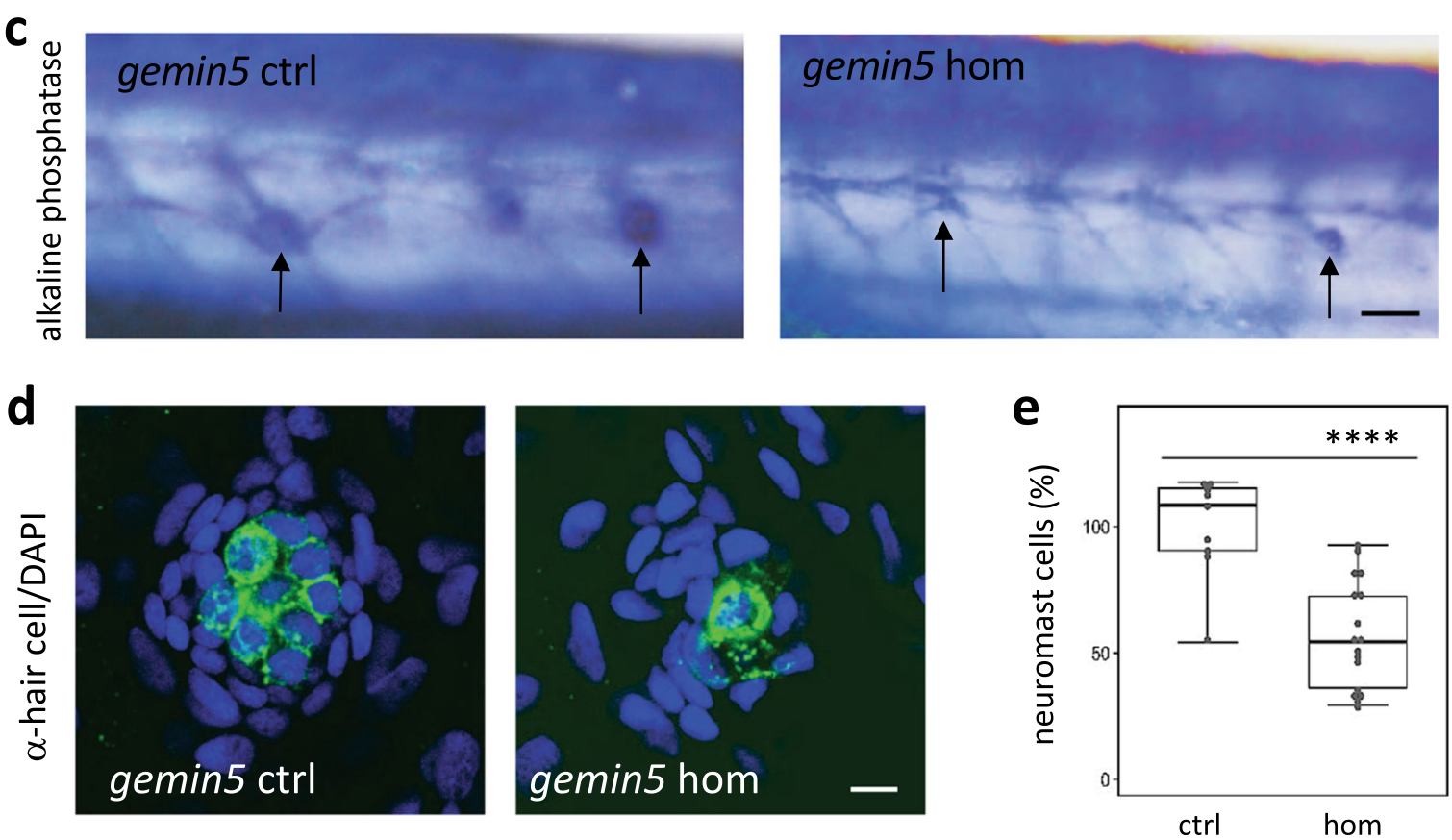

Fig. 2 gemin $5^{\text {hg81 }}$ mutants possess smaller neuromasts at 2 days post hair cell ablation. a Live cell imaging of support cells by $\mathrm{Tg}(\mathrm{tnks} 1 \mathrm{bp} 1$ : GFP) and hair cells by Tg(atoh1a:dTomato) in lateral line neuromasts of the gemin $5^{\text {hg }} 8$ control and mutant embryos at 2 days post hair cell ablation. Scale bar, $10 \mu \mathrm{m}$. b Live cell labeling of neuromast cells by $\mathrm{Tg}\left(\mathrm{cldnb}\right.$ :EGFP) in the lateral line of the gemin $5^{\text {hg81 }}$ control and mutant embryos at 2 days post hair cell ablation. Dotted white circle demarcates the periphery of the neuromast. Scale bar, $10 \mu \mathrm{m}$. c Alkaline phosphatase staining of lateral line neuromasts in the gemin $5^{\text {hg }}{ }^{81}$ control and mutant embryos at 2 days post hair cell ablation. Arrows point to the neuromasts, which are significantly smaller in the mutants when compared to those in the control larvae. Scale bar, $50 \mu \mathrm{m}$. $\mathbf{d}$ Confocal images of lateral line neuromasts in the gemin $5^{\text {hg81 }}$ control and mutant embryos at 2 days post hair cell ablation, stained with anti-hair cell antibodies (green color) and DAPI (blue color). Representative images are shown. Scale bar, $10 \mu \mathrm{m}$. e Quantification of neuromast cells. Error bars in the graphs represent mean \pm s.e.m. There is a significant reduction in the number of neuromast cells $\left({ }^{* * * *} P<0.0001\right)$. The numbers are presented as percentage because they were obtained from quantification of still confocal images. Each data point was generated from $\sim 10$ embryos.

smn1, gemin3, and gemin5 mutations affect the regeneration of multiple tissues

Regeneration of different tissues can be achieved by utilizing a common set of molecular pathways and many genes are pan- regenerative in that they are induced and essential regardless of the specific injured tissue ${ }^{21}$. Both $s m n 1$ and gemin 5 genes were involved in regulating the regeneration of multiple tissues including neuromasts, caudal fins, and livers ${ }^{14}$. To determine 
a

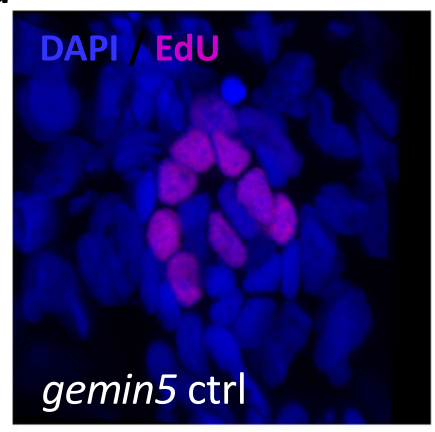

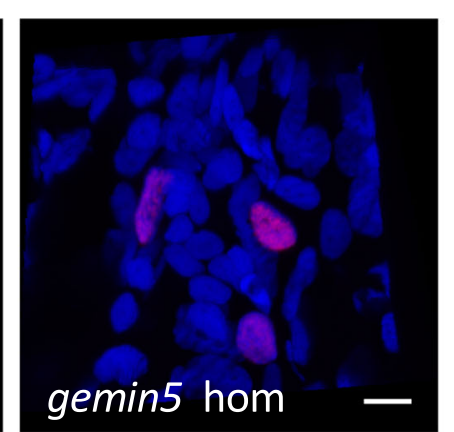

b

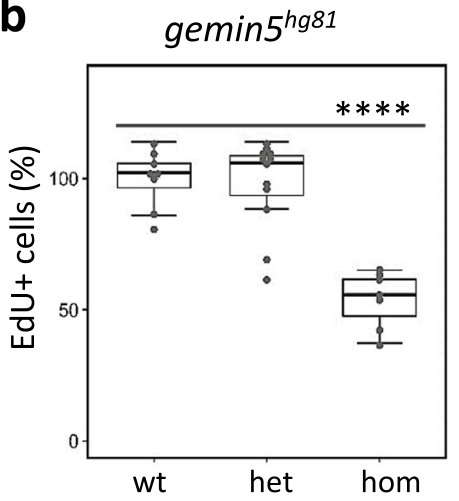

C

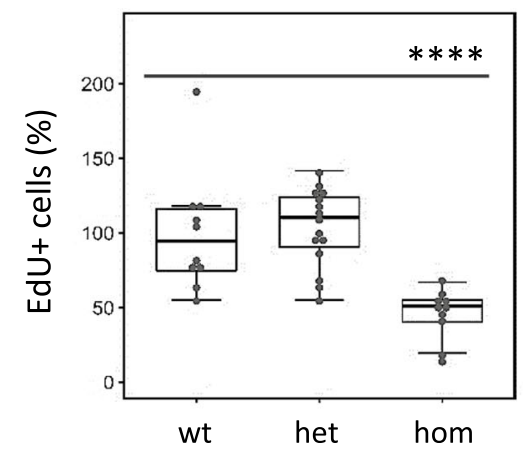

d

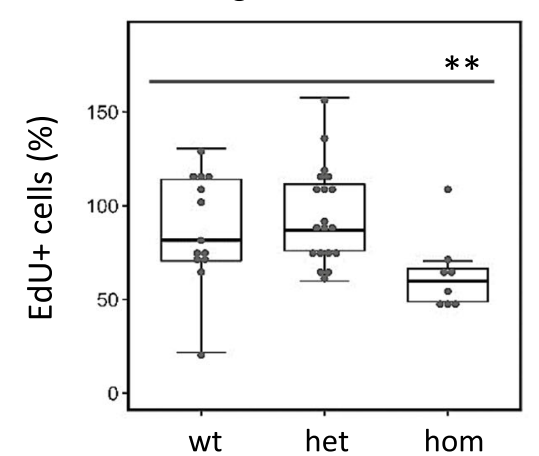

Fig. 3 Decreased neuromast cell proliferation after hair cell ablation in gemin3, gemin5, and smn 1 mutants. a Confocal images of lateral line neuromasts in the control and gemin $5^{\text {hg81 }}$ mutant at 1-day post hair cell ablation. Neuromasts were stained with DAPI (blue) and proliferating cells were labeled by EdU (pink). The embryos used for the analysis were obtained from a pairwise incross of heterozygotic parents. Hair cells were ablated at $5 \mathrm{dpf}$. EdU treatment was conducted at 1-day post ablation. Scale bar, $10 \mu \mathrm{m}$. b Quantification of EdU-positive cells in the embryos carrying wild-type, heterozygous, or homozygous gemin $5^{\text {hg } 81}$ mutations. c Quantification of EdUpositive cells in the embryos carrying wild-type, heterozygous, or homozygous $5 \mathrm{mn} 7^{\text {th229 }}$ mutations. d Quantification of EdU-positive cells in the embryos carrying wild type, heterozygous, or homozygous gemin $3^{\text {hg }} 105$ mutations. The graphs show mean \pm s.e.m. Homozygous mutants for all three regeneration genes have a significantly reduced number of proliferating cells. ${ }^{* *} P<0.01 ;{ }^{* * * * P}<$ 0.0001. Data for each mutation were generated using $\sim 40$ embryos born from a single pair of heterozygous parents.

whether gemin3 had similar phenotypes, we examined the regeneration of caudal fin and liver in gemin3 mutants. Like in smn1 and gemin5 mutants, we found gemin3 mutations did not alter the normal development of caudal fins or livers (data not shown), however, upon damage the mutant exhibited a deficiency in restoring the damaged tissues as was seen with the other mutants. After caudal fin amputation, the restored fin in the gemin3 mutant was significantly smaller and often missing the pigment gap present in normal tailfins (Fig. 4a, b). Similarly, following chemical-mediated liver ablation in the $\mathrm{Tg}$ (fabp10a:CFPNTR) transgenic background, the gemin3 mutant displayed a clear deficiency in liver regeneration compared to the control siblings (Fig. 4c, d). As a control, gemin6 mutants were also examined for a role in the regeneration of caudal fin and liver. In contrast to the regeneration mutants, gemin6 mutants showed normal regeneration of both the caudal fin and the liver (Supplementary Fig. 5). These data suggest that gemin3, like $s m n 1$ and gemin5, is generally involved in regeneration, regardless of the injured tissue.

RNA-Seq reveals shared downstream targets among the genes involved in regeneration

To identify the pathways shared amongst the mutants blocking regeneration, we conducted RNA-Seq using all the mutants from the SMN complex that affected adult survival. We found that smn1, gemin3, and gemin5 clustered together while all of the mutations not resulting in a regeneration phenotype failed to cluster with any other gene (Fig. 5a), suggesting the function of the genes without a regeneration phenotype are more divergent than that of the regeneration genes. The regeneration genes regulated a common set of downstream targets which were distinct from the mutants that did not affect regeneration (Supplementary Fig. 6, Supplementary Data 1). We found erbb2 and erbb3b, two genes with a documented role in neuromast hair cell formation ${ }^{28,29}$, were upregulated in the three nonregenerative mutants (Fig. 5b, c). RNA-Seq data also identified an increase in expression for tp53 and $m d m 2$ genes specifically in the mutants inhibiting regeneration (Supplementary Fig. 7a). In addition, RNA-Seq data showed that a mutation in one of the "regeneration genes" had no effect on the expression of the other two genes (Supplementary Fig. 8), suggesting there is no interregulation between the genes at the transcriptional level.

Observed upregulation of the tp53/Mdm2 pathway was not the major cause of the regeneration phenotype

Several lines of published evidence indicate that p53 interacts with and regulates $\mathrm{Mdm} 2$, and activation of tp53 is associated with SMN complex activity and $S_{M A}{ }^{30-32}$. Our RNA-Seq data showed a regeneration-associated upregulation of both the tp53 and $m d m 2$ genes (Supplementary Fig. 7a). To investigate a potential role for the tp53/Mdm2 pathway in hair cell regeneration, we depleted tp53 
a
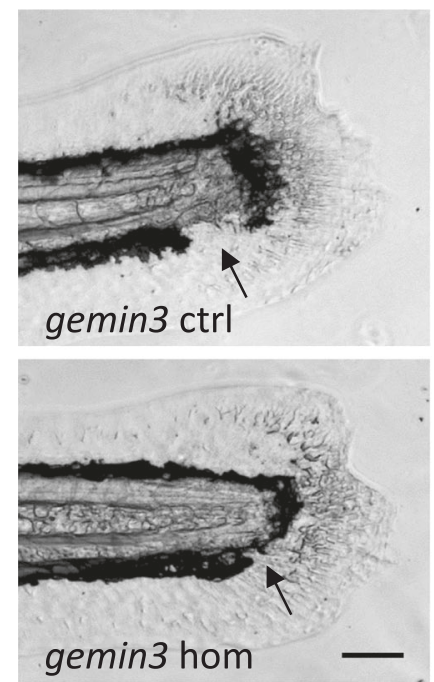

C
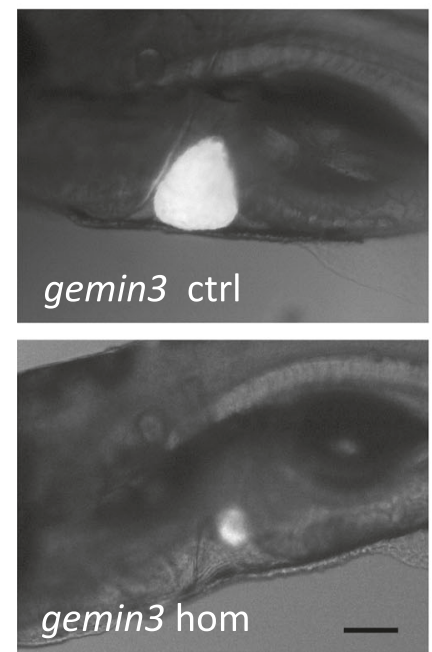

b

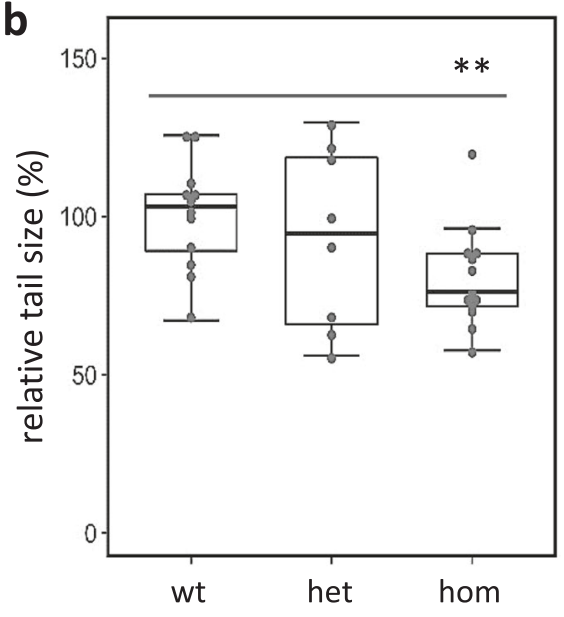

d

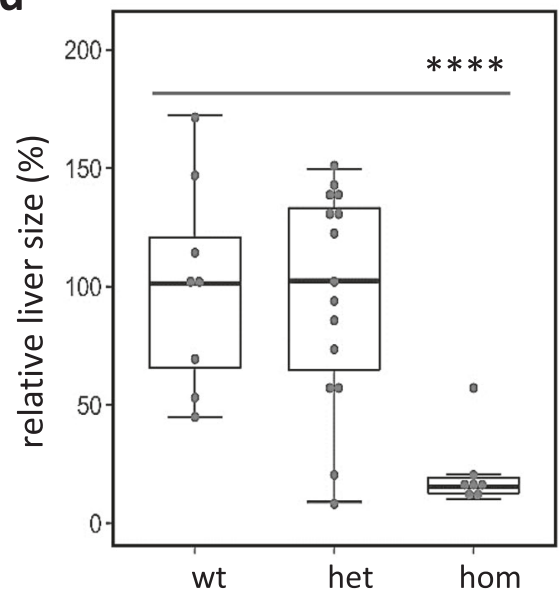

Fig. 4 gemin $3^{\text {hg }}{ }^{106}$ mutations impair regeneration of caudal fins and livers. a Caudal fin regeneration in the control and gemin $3^{\text {hg } 106}$ mutant embryos at 4 days post amputation. Arrows point to the pigment gap which is often missing in the mutants. Scale bar, $100 \mu$ m. b Quantification of the area of regenerated caudal fins in the gemin $3^{\text {hg } 106}$ mutants. c Liver regeneration in the control and gemin $3^{\text {hg }}{ }^{106}$ mutant embryos at 3 days post ablation. The CFP fluorescence labels the regenerated livers. Scale bar, $100 \mu$ m. $\mathbf{d}$ Quantification of the area of the regenerated livers. Representative images are shown. Liver tissue is labeled by Tg(fabp10a:CFP-NTR). Graphs show mean \pm s.e.m. ${ }^{* * P}<0.01$; $* * * * P<0.0001$. Data for each analysis were collected from $\sim 40$ embryos produced from a pairwise heterozygous incross (for fin regeneration), or a pairwise heterozygous incross in the background of the transgene $\mathrm{Tg}(f a b p 10 a$ :CFP-NTR) (for liver regeneration).

genetically in both the smn1 and gemin5 mutant backgrounds. For the gemin5 study, we crossed the gemin 5 mutant with the tp53 M214K mutation ${ }^{33}$, and found that the gemin5/tp53 double mutants showed no improvement in regeneration or adult survival when compared to the gemin 5 mutant alone (Supplementary Fig. 7b, data not shown). For the smn1 mutation, the tp53 and smn1 genes in zebrafish were both on chromosome 5 , so we obtained double mutants carrying homozygous mutations for an smn 12 bp insertion and a tp53 7 bp deletion, by injecting smn1 CRISPR guide RNAs into embryos harboring a homozygous 7 bp tp53 deletion mutation ${ }^{34}$ and raised those fish for inbreeding. Consistent with the results of the gemin5/tp53 double mutant, tp53 mutants did not rescue the regeneration deficiency or the adult survival of $s m n 1$ mutants (data not shown).

RNA-Seq data showed that Mdm2 was also significantly induced in gemin3, gemin5, and smn1 mutants. Since mdm2 mutations cause early embryonic lethality, we created a partial knockdown of Mdm2 by injecting $m d m 2$ CRISPR guide RNAs into the gemin 5 mutant background and found the resulting mosaic mutations in $m d m 2$ showed no rescue of hair cell regeneration in the gemin5 mutants (data not shown). Altogether, these data indicate that despite strong induction of $t p 53$ and $m d m 2$ in all three mutants blocking regeneration, the tp53/Mdm2 pathway is not a major contributor to the observed regeneration phenotype although it does suggest that all three genes are involved in a common subset of pathways not shared by the other SMN complex proteins, and those pathways are involved in both injury responses and tp53 stress responses.

Regeneration-deficient mutants are less sensitive to the ErbB pathway inhibitor AG1478

RNA-Seq analysis revealed an upregulation of erbb3b in the three regeneration-deficient mutants (Fig. $5 b$ ). To investigate whether the ErbB pathway was associated with the hair cell regeneration deficiency, we performed a pharmacological inhibition using a well-established ErbB pathway inhibitor, AG1478, on gemin5 mutants. Since the RNA-Seq data were obtained from analyzing 
a

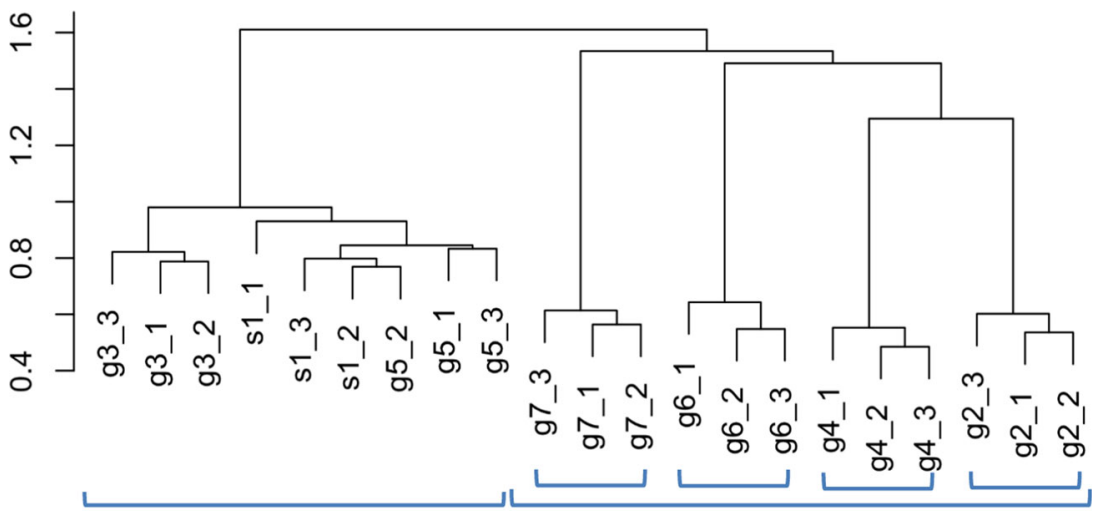

regeneration mutants

non-regeneration mutants

b

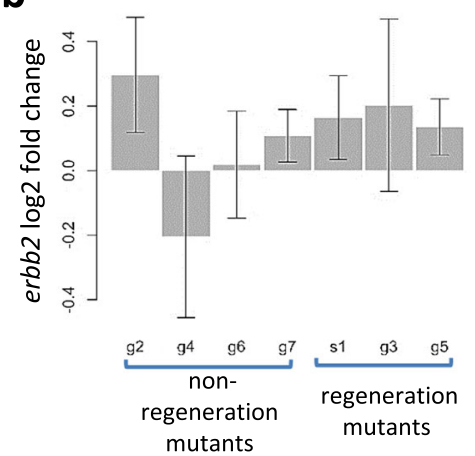

C

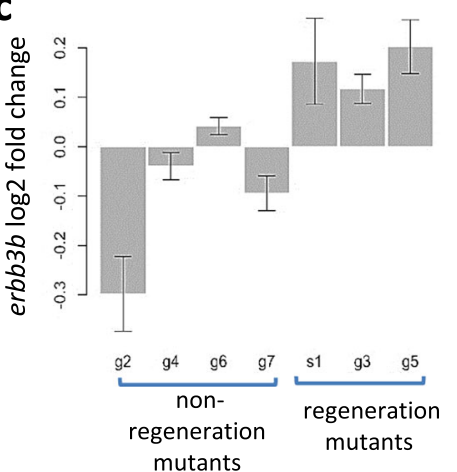

Fig. 5 Shared genes dysregulated in gemin3, gemin5, and smn 1 mutants revealed by RNA-Seq. a Hierarchical clustering of RNA-Seq samples using log2-fold change of normalized read counts. The control and mutant embryos used for the analysis were: $s m n 1^{\text {fh22 }}$ (s1),

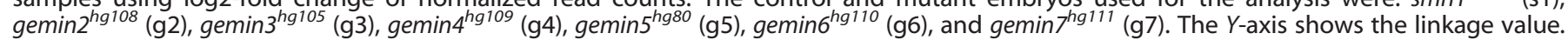
b and c Log2-fold change of mRNA expression of erbb2 b and erbb3b c in different homozygous mutant backgrounds. Error bars indicate standard deviations.

whole larval tissue and the regeneration phenotypes were manifested in the lateral line neuromasts, we examined the impact of perturbating other pathways that were enriched in the RNA-Seq data analysis and/or have a documented role in neuromast cell proliferation (Supplementary Table 3). Most conditions were negative, with only AG1478 showing a specific phenotype. Treatment with $2 \mu \mathrm{M}$ AG1478 caused a dramatic increase in lateral line neuromasts of control siblings (as predicted), but only a mild increase in the gemin5 mutant larvae (Fig. 6a, b, Supplementary Fig. 9a), indicating that the gemin5 mutant is resistant to ErbB pathway inhibition. To determine whether the reduced sensitivity was common to all three regeneration gene mutations, we treated smn1 and gemin3 mutants with AG1478. Consistent with the findings from the gemin 5 mutant, the smn 1 mutant and the gemin 3 mutants also showed a reduced responsiveness to AG1478 (Fig. 6c, d). To test if the ErbB pathway responded normally in the other mutants of the complex, we treated gemin6 mutants with AG1478. In contrast to the mutants that disrupted regeneration, the gemin6 mutant responded to AG1478 comparable to that of their control siblings (Fig. 6e). Altogether, these results point out that the inability to respond to AG1478 inhibition specifically in the mutants that inhibited regeneration, suggesting a mechanistic link between the loss of regeneration and ErbB signaling.

We observed a down-regulation of erbb3b and an upregulation of erbb2 in the gemin2 mutant (Fig. 5b, c). To examine whether ErbB signaling is altered in gemin2 mutants, we analyzed the neuromast formation under natural conditions and under ErbB pathway inhibition. Neither condition revealed a difference between the control siblings and gemin2 mutants (Supplementary Fig. 9b), suggesting ErbB signaling in gemin2 mutant is normal.

Neuromast cell proliferation is not induced by AG1478 in gemin5 mutants

To understand why gemin5 mutants responded differently to AG1478, we used wild-type embryos with a double transgene $\mathrm{Tg}$ (pou4f3:GAP-GFP);(SqET20:EGFP) to label neuromast cells, and used an EdU incorporation assay to mark proliferating cells. We exposed the double transgenic embryos either to a mock treatment or to AG1478, and the resulting embryos were stained with the nuclear dye DAPI. In each neuromast, DAPI labeled all neuromast cells, $\mathrm{Tg}$ (pou4f3:GAP-GFP) labeled hair cells, Tg(SqET20:EGFP) labeled mantle cells that demarcate the outer periphery of neuromasts, and the GFP negative and DAPI-positive cells in between were the support cells. Quantification results showed that AG1478 promoted the proliferation of all three types of neuromast cells in wild-type larvae (Supplementary Fig. 9c, d), consistent with previous finding that AG1478 promotes cell proliferation ${ }^{35}$.

When AG1478 was applied to gemin5 embryos possessing the same Tg(pou4f3:GAP-GFP);(SqET20:EGFP) transgenes, gemin5 mutants possessed a significantly reduced number of neuromast hair cells (as visualized by $\mathrm{Tg}($ pou4f3:GAP-GFP)) and mantle cells (as visualized by $\mathrm{Tg}(\mathrm{SqET} 20: \mathrm{GFP})$ ) (Fig. 6f), indicating that the gemin5 mutation impaired neuromast cell proliferation in response to ErbB inhibition.

Several studies have indicated that AG1478 regulates neuromast cell proliferation through modulating the cell signaling activity between the Schwann cells, interneuromast cells, and the axons via $\mathrm{Wnt}^{35-38}$. We tested whether we could detect 
a
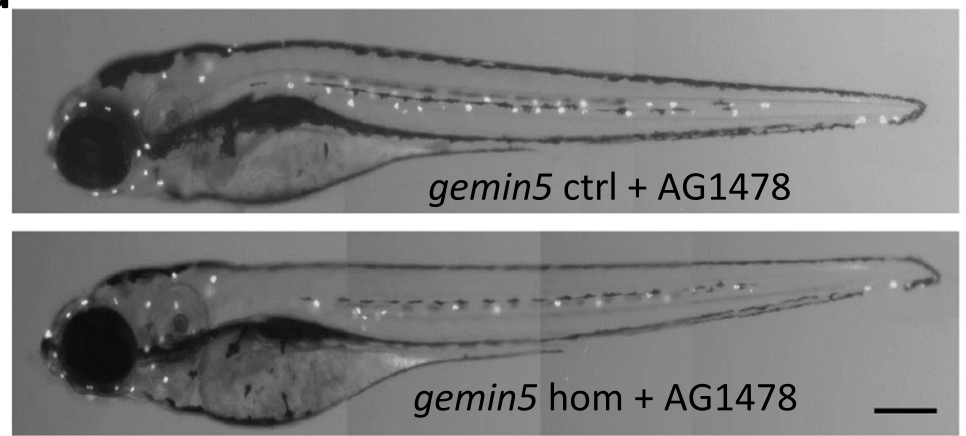

C

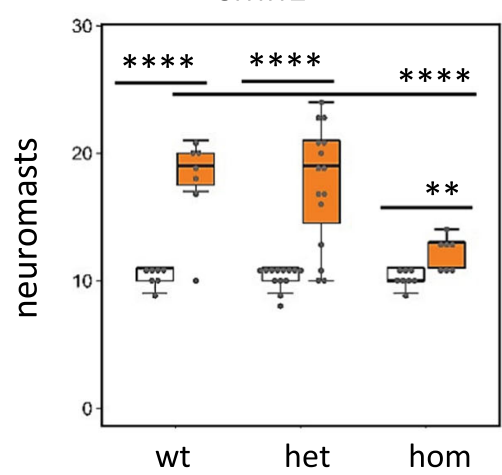

d $\operatorname{gemin} 3^{\text {hg } 105}$

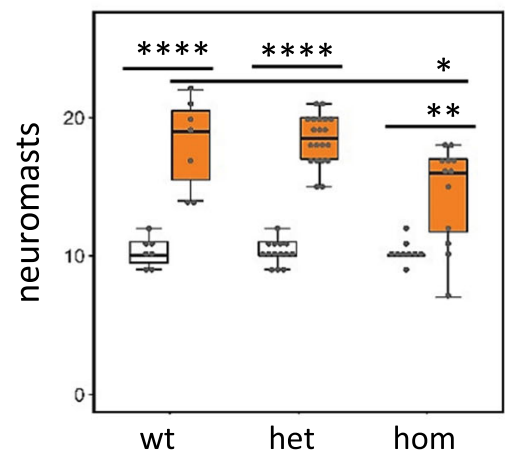

b

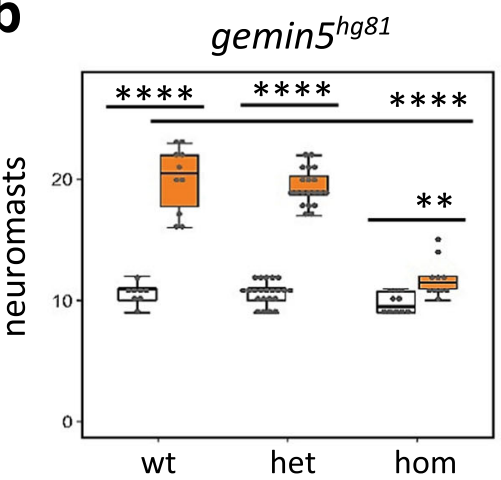

e

gemin $6^{h g 110}$

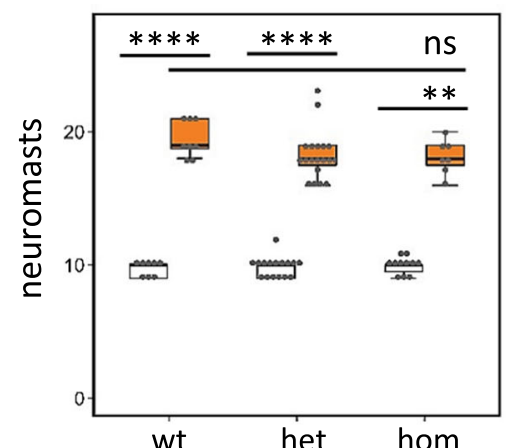

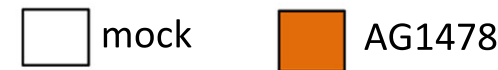

f
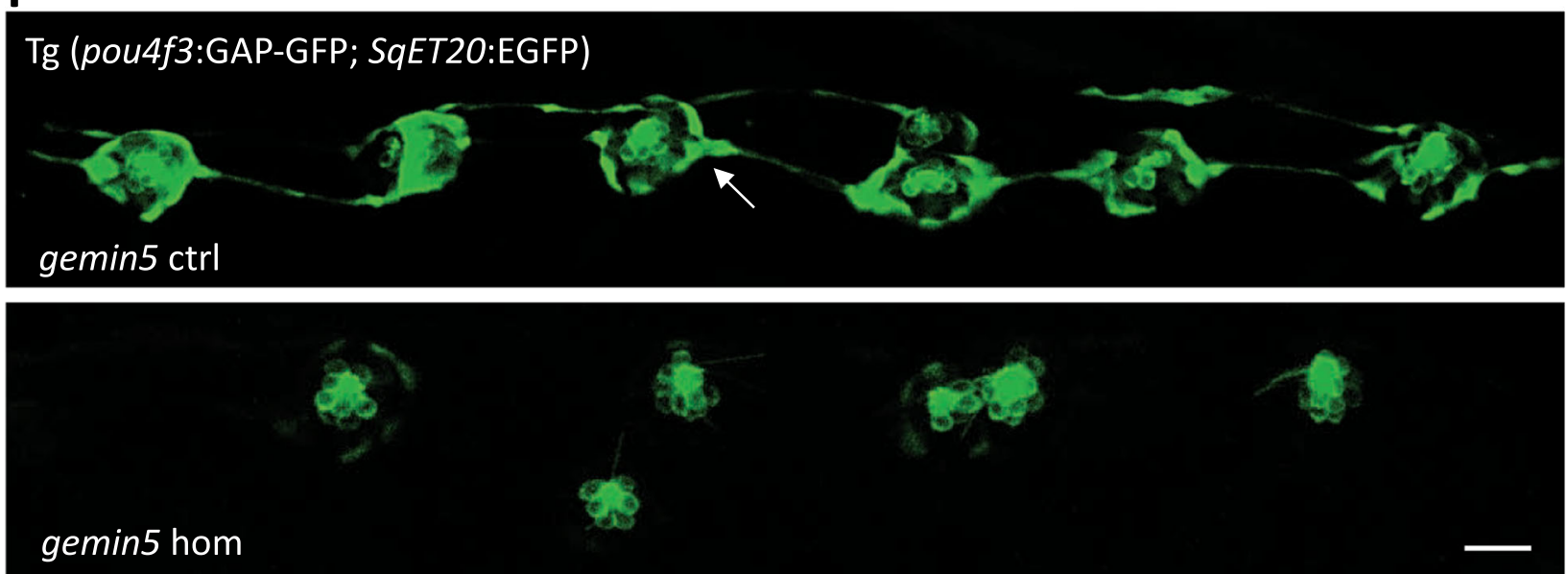

Fig. 6 Reduced responsiveness of gemin3, gemin5, and smn 1 mutants to ErbB pathway inhibitor AG1478. a Neuromasts in AG1478-treated control and gemin $5^{\text {hg81 }}$ mutant embryos at 5 dpf. Neuromasts are shown as white dots. Scale bar, $250 \mu \mathrm{m}$. b Quantification of the lateral line neuromasts in mock and AG1478-treated embryos carrying the gemin $5^{\text {hg } 81}$ mutation at 5 dpf. c Quantification of the lateral line neuromasts in mock and AG1478-treated embryos carrying the $s m n 1^{\text {hg }} 104$ mutation at $5 \mathrm{dpf}$. d Quantification of the lateral line neuromasts in mock and AG1478treated embryos carrying the gemin $3^{\text {hg105 }}$ mutation at $5 \mathrm{dpf}$. e Quantification of the lateral line neuromasts in mock and AG1478-treated embryos carrying the gemin $6^{h g 110}$ mutation at 5 dpf. Error bars in the graphs show the mean \pm s.e.m. ns, $P>0.05 ;{ }^{*} P<0.05 ;{ }^{* *} P<0.01 ;{ }^{* * * *} P<0.0001$. f Fluorescent images of lateral line neuromasts labeled by transgenes Tg(pou4f3:GAP-GFP) and Tg(SqET20:EGFP) in the control and gemin $5^{\text {hg }}$ i mutant at $5 \mathrm{dpf}$ after AG1478 treatment. Images were taken in the areas surrounding the end of yolk extension. White arrow points to the Tg (SqET20:EGFP) signal in the control embryo, which is dramatically increased in the mutant. Scale bar, $50 \mu \mathrm{m}$. The embryos used for the above

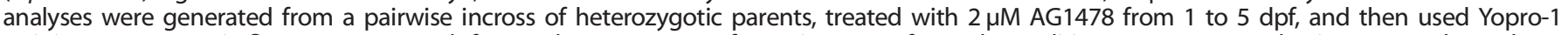
staining or transgenic fluorescence at $5 \mathrm{dpf}$ to analyze neuromast formation. Data for each condition were generated using $\sim 40$ embryos born from a single pair of heterozygous parents. 
disruptions in Schwann cells in gemin5 mutant embryos. Schwann cell morphology and quantity were evaluated using the $\operatorname{Tg}$ (foxd3: GFP) transgene or by the expression of myelin basic protein a $(m b p a)$. Neither revealed a noticeable difference between the control siblings and mutants (Supplementary Fig. 10a, b). Lateral line axons were labeled with an antibody targeting acetylated tubulin and appeared to be comparable between the control and mutant embryos (Supplementary Fig. 10c). Wnt pathway activity was manipulated with both the Wnt pathway activator BIO and the inhibitor IWR1. Neither showed any differences between the mutant and control siblings (Supplementary Table 3). Altogether these data suggest the disruptions in myelination by the Schwann cells was not associated with the failure of AG1478 to induce supernumerary neuromasts in the gemin 5 mutant.

Genetic mutations of ErbB pathway genes recapitulate the AG1478 effect

Similar to inhibition by AG1478, mutations in ErbB pathway genes, such as erbb2, erbb3b, and $n r g 1$, lead to an increase in lateral line neuromasts ${ }^{35,37,38}$. Mutations in erbb3b and $n r g 1$ appear to have no other significant impact on embryo axis patterning nor on adult survival. We therefore generated stable genetic mutations for both $e r b b 3 b$ and $n r g 1$ and compared the effect of these mutations to the AG1478 effect on lateral line neuromast formation. As expected, both erbb3b and nrg1 mutations caused a dramatic increase in the number of lateral line neuromasts, however, the increase was consistently lower than that of AG1478 treatment (Fig. 7a, b), suggesting AG1478 inhibits ErbB signaling more broadly than that mediated by either erbb3b or $n r g 1$ alone and that there may be some redundant signaling from other related genes.

We then examined how mutations of the genes in the ErbB pathway impact neuromast formation in the gemin5 mutant background. We generated double mutants of erbb3b/gemin5 or nrg1/gemin5. Consistent with our previous observations, a homozygous mutation in either erbb3b or nrg1 alone caused an increase in the number of neuromasts, a homozygous mutation for gemin5 alone caused no alteration, and a heterozygous mutation of either gene alone or together produced no change (Fig. 7c, d). Both double mutants displayed a lower level of increase of lateral line neuromasts when compared to erbb3b or nrg1 mutant, however, the number of neuromasts in the double mutants was significantly higher when compared to the gemin5 mutant alone, indicating disruption of the ErbB pathway could partially rescue the deficiency of neuromast formation in the mutant. The partial rescue in the double mutants suggests that AG1478 was failing to sufficiently inhibit ErbB signaling in gemin5 mutants instead of the alternative explanation that interneuromast cells were unable to respond properly to release of ErbB signaling.

Rescue was also attempted by mutating the erbb2 gene in the gemin 5 mutant background. Since erbb2 loss of function is early embryonically lethal, we generated a mosaic knockdown of erbb2 by injecting multiple CRISPR guide RNAs into the embryos from a gemin 5 heterozygous incross, and then used the injected embryos to quantify lateral line neuromast formation. Mutation frequency analysis showed these erbb2 CRISPR guide RNAs resulted in a nearcomplete mutagenesis of the erbb2 gene (Fig. 7e). Neuromast number quantification showed the erbb2 knockdown promoted more neuromasts than that of gemin5 mutant (Fig. 7f).

ErbB pathway inhibition partially rescues hair cell regeneration Activation of ErbB signaling has been implicated in the regeneration of other tissues ${ }^{29,39}$, so we investigated whether ErbB pathway inhibition could improve hair cell regeneration in the three mutants that disrupt regeneration. In performing the hair cell regeneration assay in the presence of the ErbB inhibitor, AG1478 had no obvious effect on the regeneration of hair cells in control siblings, however, it did exhibit a dose-dependent rescue of regeneration in all three mutants (Fig. 8). Our interpretation of the data from Figs. 7 and 8 is that ErbB signaling in the smn1, gemin3, and gemin5 mutants was hyperactive, such that the increased ErbB activity was blocking AG1478 induction of ectopic neuromasts. Similarly, too much ErbB signaling was blocking the initiation of hair cell regeneration, but now AG1478 inhibition was sufficient to partially release the block in regeneration, presumably because ErbB-signaling levels were generally lower in the regenerating neuromast compared to the interneuromast cells, or the level of reduction needed to see rescue was lower in the case of hair cell regeneration compared to neuromast induction.

\section{DISCUSSION}

Our previous large-scale mutagenesis screen showed smn1 and gemin5, two SMN complex members, were essential for tissue regeneration ${ }^{14}$. In this study, we expanded our mutagenesis screen to systematically examine the potential role of each of the nine members of the SMN complex in tissue regeneration. Consistent with the findings reported from other groups ${ }^{40-42}$, we found that mutations in most SMN complex members were essential for adult survival (Supplementary Table 2). However, our genetic data suggest the nine SMN complex members can be categorized into three separate groups: smn1, gemin3, and gemin5 are required for both overall survival and regeneration after injury; gemin2, gemin4, gemin6, and gemin7 are required for survival but not for regeneration; gemin8 and strap/unrip appear to be non-essential for either regeneration or survival. The three regeneration members (smn1, gemin3, and gemin5) are regulating regeneration through ErbB pathway-mediated cell proliferation, and they are essential for regeneration of multiple (if not all) tissues.

Studies of the SMN complex have been ongoing for more than two decades ${ }^{43}$ with the largest focus on SMN1 because mutations in this gene are responsible for the human disease $\mathrm{SMA}^{1,2}$. However, the association of SMN complex members with tissue regeneration was not recognized until our prior study ${ }^{14}$ and expanded here. Our work strongly suggests that some of the SMN complex members have separate, independent functions unrelated to snRNP assembly, or that the complex may not have a single cellular function that always requires all nine subunits in stochiometric balance. For example, we found transcripts were not expressed uniformly and ubiquitously, but expression varied in different brain regions and in different neuromast cells (Supplementary Fig. 2). However, no additive or synergistic interactions were observed between the three genes involved in regeneration (Supplementary Fig. 3). All three regeneration members functioned through ErbB-pathway-mediated cell proliferation (Figs. 6, 8), all three possessed an ability to regulate regeneration in multiple tissues (Fig. 4) ${ }^{14}$, and none of the three appeared to be epistatic to the other two. All these findings argue that these regenerative members work together in a shared molecular mechanism. Our findings suggest that the three SMN complex members involved in regeneration possess functions separate from snRNP biosynthesis that are essential for tissue regeneration and are also related to tp53 regulation/activation, although our genetic evidence in these two functions are not directly related.

In line with our findings, previous studies have reported apparently independent activities of SMN complex members. For example, SMN1 regulates ribosome biology and motor neuron growth ${ }^{5,44,45}$, SMN1's function in motor neurons appears to be independent of snRNP biosynthesis ${ }^{46}$, and SMN1 has a specific role in axonal mRNA regulation and axonogenesis ${ }^{7,8}$. Furthermore, GEMIN3, an RNA helicase, is involved in cell proliferation and microRNA regulation of signal transduction ${ }^{9}$. Gemin5 regulates $s m n 1$ expression ${ }^{25}$, Gemin5's C-terminus can regulate protein synthesis ${ }^{10,12,47,48}$, and Gemin5 is strongly stimulated upon viral infection ${ }^{11}$. Future studies should be able to evaluate SMN complex-dependent and independent functions more precisely through detailed analysis of splicing isoforms 

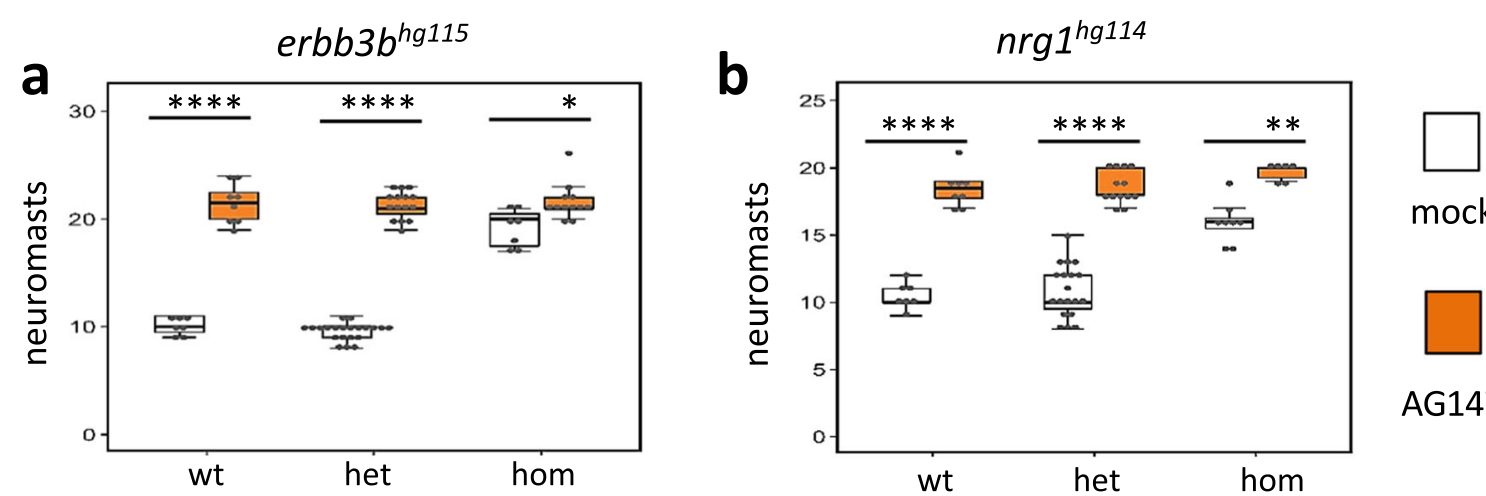

mock

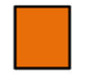

AG1478
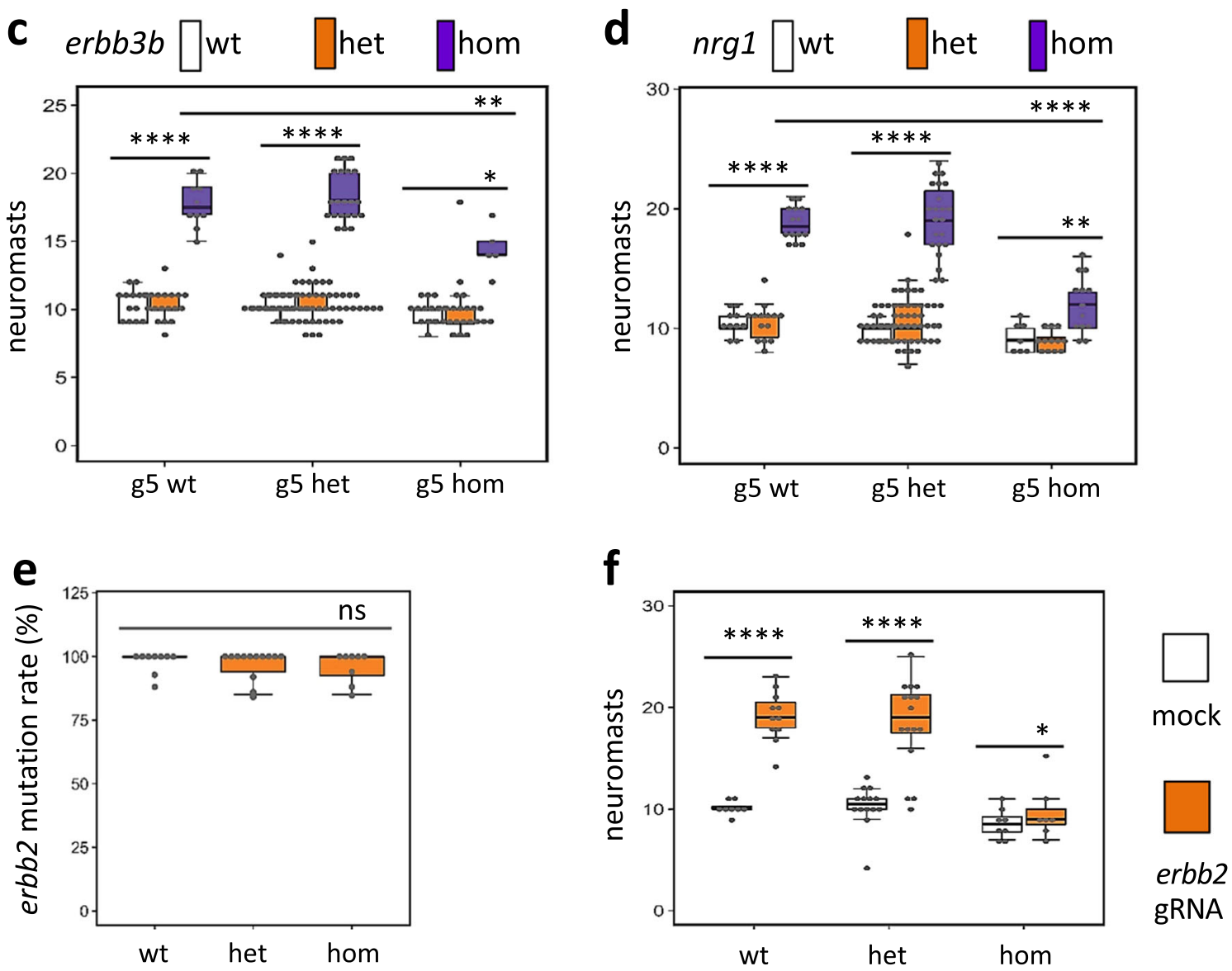

Fig. 7 Reduced responsiveness of gemin $^{\text {hg81 }}$ mutants to the knockdown of ErbB pathway genes. a Quantification of lateral line neuromasts in the mock and AG1478-treated erbb3b $b^{\text {hg } 115}$ mutant embryos at $5 \mathrm{dpf}$. b Quantification of lateral line neuromasts in the mock and AG1478-treated $\mathrm{nrg} 1^{\mathrm{hg}} 114$ mutant embryos at $5 \mathrm{dpf}$. Approximately 40 embryos generated from a pair of heterozygous parents carrying either

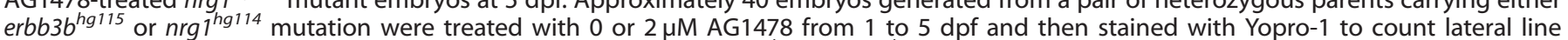
neuromasts. c Quantification of lateral line neuromasts in the gemin $5^{\text {hg } 81} /$ erbb $3 b^{\text {hg } 115}$ mutant embryos at 5 dpf. The data are generated from analyzing 177 embryos generated from pairwise incrosses of double heterozygous parents and five embryos are double mutants. d Quantification of lateral line neuromasts in the gemin $5^{\text {hg81 }} / \mathrm{nrg} 1^{\text {hg }}{ }^{114}$ mutant embryos at 5 dpf. The data are generated from analyzing 156 embryos generated from pairwise incrosses of double heterozygous parents and 13 embryos are double mutants. e erbb2 mutation rate in erbb2 CRISPR guide RNA injected gemin5 mutant embryos at $5 \mathrm{dpf}$. Mutation rate was measured by CRISPR-STAT fluorescent PCR-based fragment analysis ${ }^{61}$. $\mathbf{f}$ Quantification of lateral line neuromasts in the mock and erbb2 CRISPR guide RNA injected gemin 5 mutant embryos at 5 dpf. Error bars in the graphs indicate mean \pm s.e.m. ns, $P>0.05 ;{ }^{*} P<0.05 ; * * P<0.01 ; * * * P<0.0001$. The analysis was done by injecting the embryos generated from a pair of gemin $5^{h g 81}$ heterozygous parents with either Cas9 protein alone (mock injection) or Cas9 protein together with erbb2 guide RNAs (erbb2 gRNA injection), followed by analyzing hair cell development in $\sim 40$ injected embryos for each condition, and lastly determining gemin $5^{\text {hg } 81}$ genotype and erbb2 mutation rate for each of the analyzed embryo. No erbb2 mutation was detected in the mock injection group. 
a

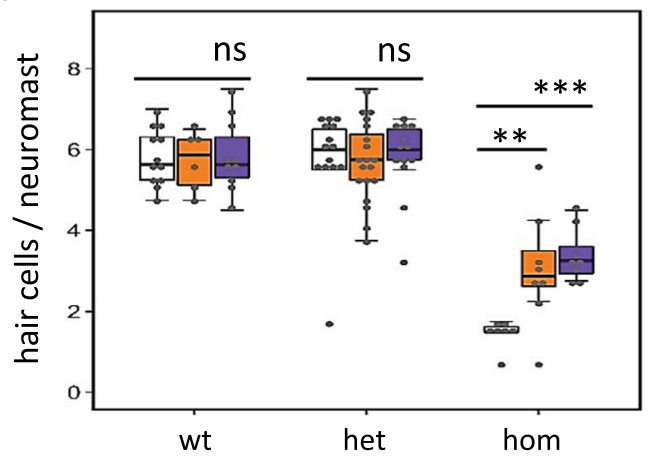

b

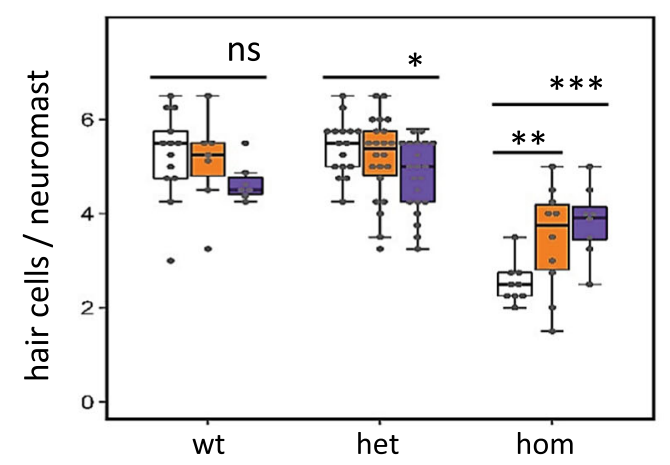

C

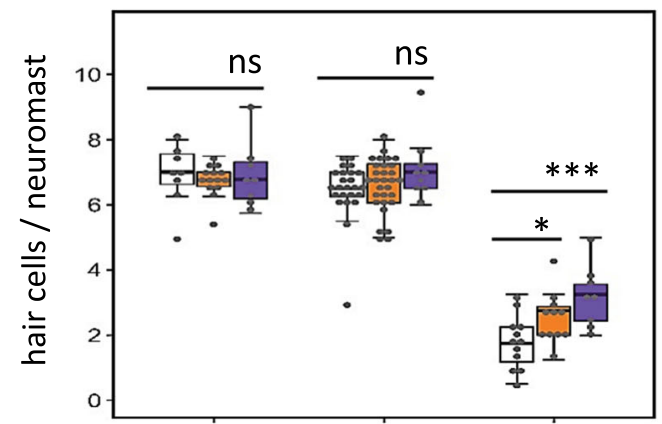

$\square$ mock $2.5 \mu \mathrm{M} \mathrm{AG} 1478 \square 5 \mu \mathrm{M}$ AG1478

Fig. 8 ErbB pathway inhibitor AG1478 partially rescues the hair cell regeneration deficiency in gemin3, gemin5, and smn 1 mutants. a Quantification of regenerated hair cells in the AG1478treated $s m n 7^{\text {hg }} 104$ mutant embryos at 2 days post hair cell ablation. b Quantification of regenerated hair cells in AG1478-treated gemin $3^{\text {hg } 105}$ mutant embryos at 2 days post hair cell ablation. c Quantification of regenerated hair cells in lateral line neuromasts in AG1478-treated gemin $5^{\text {hg81 }}$ mutant embryos at 2 days post hair cell ablation. The slight reduction in the hair cells of the gemin $3^{\text {hg } 105}$ heterozygotes treated with $5 \mu \mathrm{M}$ of AG1478 could be due to drug toxicity to this genetic background, since it was not observed in the smn $1^{\text {hg } 104}$ and gemin $5^{\text {hg }}{ }^{17}$ embryos. Graphs show the mean \pm s.e.m. Statistical difference are indicated as: ns, $P>0.05$; ${ }^{*} P<0.05 ;{ }^{* *} P<$ $0.01 ; * * * P<0.001$. Analysis for each condition was done with $\sim 40$ embryos generated from a single pair of heterozygous carrier parents, ablated hair cells at $5 \mathrm{dpf}$, and then treated with $0,2.5$, or $5 \mu \mathrm{M}$ of AG1478 from 5 to $7 \mathrm{dpf}$. in different genes and/or cell types, under natural, diseased, or regenerative conditions.

In this study, we demonstrated a link between the ErbB pathway and three of the SMN complex's proteins. RNA-Seq data revealed an increased expression of the ErbB pathway genes erbb2 and erbb3b in smn1, gemin3, and gemin5 mutants (Fig. 5b, c), both chemical inhibition of the ErbB pathway with AG1748 and genetic ablation of erbb3b or nrg1 were able to partially rescue the neuromast induction and hair cell regeneration (Figs. 6-8), demonstrating the ErbB pathway was hyperactive in the mutants. Because the ErbB pathway is associated with various neurological diseases ${ }^{49}$, it suggests future investigation is warranted to address whether the upregulation of the ErbB pathway in the three SMN mutants is specific to injury responses or if it is also one of the underlying mechanisms in the neurodegenerative pathology of SMA.

Several studies have demonstrated that the ErbB pathway plays a promotive role in the regeneration of other tissues. For example, mutations in erbb2 or erbb3 cause a deficiency in caudal fin regeneration ${ }^{29}$, and AG1478 treatment inhibits the regenerative proliferation of cardiomyocytes ${ }^{39}$. Our data indicate that the role of the ErbB pathway in regeneration differs based on tissue type. It remains unclear how the ErbB signaling is properly integrated into the different roles it plays in different tissues.

We found that inhibition of ErbB pathway contributes to a partial rescue of their regeneration phenotype (Fig. 8). The partial rescue, consistent with the RNA-Seq data (Fig. 5. Supplementary Fig. 6), suggests that the ErbB pathway is, at least in part, the underlying mechanism for the deficient regeneration, and likely it is only one of many pathways affected during the regeneration. It is possible that this dysregulation of ErbB signaling is also responsible for some of the pathologies seen in SMA patients, suggesting that one possible treatment could be inhibitors of ErbB signaling. Besides erbb3b, p53, and $m d m 2$ were also upregulated (Supplementary Fig. 7a). The p53/Mdm2 pathway has been documented to interact with the SMN complex. tp53 has a direct physical interaction with both SMN1 and Gemin3 ${ }^{32,50}$ and tp53 depletion rescues $m d m 2$ mutant phenotypes ${ }^{30}$. Abnormal $m d m 2$ splicing and p53 activation are associated with the death of motor neurons in $\mathrm{SMA}^{31}$. We found inhibition of the tp53/Mdm2 pathway brought no alteration to survival or regeneration in the gemin 5 or smn 1 mutants (Supplementary Fig. 7, data not shown), suggesting this pathway is not the major cause of the mutant regeneration phenotypes.

Despite the well-established role of the SMN complex in premRNA splicing, the number of mis-splicing events detected in the RNA-Seq dataset were relatively limited (Supplementary data 2). It suggests that depleting a single member of the SMN complex does not fully abolish the complex activity. Consistent with this idea, the formation of sub-complexes containing some but not all SMN complex members has been observed ${ }^{51,52}$. The RNA-Seq samples were collected at $7 \mathrm{dpf}$, a time prior to the manifestation of obvious morphological phenotypes. The significant regeneration deficiency detected at this time in the mutants of the three SMN complex members argues that the regeneration phenotype is not solely attributed to the splicing function of the SMN complex, in line with the observation that knockdown of the PRMT5 complex members had no effect on regeneration (Supplementary Fig. 3d, e). Studies on splicing genes and their associated human diseases have brought forth clear evidence on the noncanonical role of splicing factors in translation regulation $^{53-55}$. Taken into consideration that both SMN1 and GEMIN5 have a documented role in translational control and GEMIN5 has been shown to regulate $s m n 1$ expression ${ }^{5,10,25}$, it is reasonable to speculate that the regeneration phenotype of the three SMN complex members, even the disease SMA, could be associated with post-transcriptional roles of these proteins. Supporting this speculation is that SMA is also associated with mutations in uba1, a gene involved in protein ubiquitination ${ }^{56,57}$. 
In conclusion, this study provides insight into the SMN complex and potential roles for the complex in wound healing and ErbB signaling. Although SMN1 is the causative gene in the majority of SMA patients, there are still cases of SMA where the causative gene is unknown. Because we see phenotypes cluster with smn1, gemin3, and gemin5, it is possible that a fraction of undiagnosed SMA cases or related neurodegenerative diseases could be caused by variants in either GEMIN3 or GEMIN5. It is also possible that the functions of the three SMN complex members outside of snRNP assembly are somehow linked to SMA pathology and deficient regeneration is an underlying mechanism for SMA and even for other neurological diseases.

\section{METHODS}

\section{Zebrafish husbandry and embryology}

Zebrafish husbandry and embryo staging were performed according to $\mathrm{Kimmel}^{58}$. All experiments were in compliance with NIH guidelines for animal handling and research and approved by the NHGRI Animal Care and Use Committee (protocol G-01-3). Adult fish survival was examined at 3 months post fertilization. Quantitative PCR (qPCR) was conducted by extracting total RNA with Trizol (Invitrogen, Cat\#: 15596026), synthesizing CDNA with SuperScript first-strand synthesis system (Thermo Fisher Scientific. Cat\#: 11904018), and then running qPCR with SYBR ${ }^{\text {TM }}$ Green PCR Master Mix (Thermo Fisher Scientific, Cat\#: 4344463). Beta-actin was used as an internal reference. Semi-qPCR analysis was conducted similarly as GPCR except no use of SYBR Green and amplicons analyzed on an agarose gel. CRISPR/Cas9 mutagenesis was performed as previously described ${ }^{59}$. For studying the effect of erbb2, gemin5, clns $1 a, w d r 77$, and prmt5 knockdown on regeneration, Cas 9 protein (New England Biolabs. Cat\#: M0646T) was co-injected with multiple guide RNAs targeting a single exon of each gene to increase mutation frequency. Mutation rate was detected by a pair of primers flanking the guide RNA targets. The CRISPR targets and primers used for mutation detection are listed in the CRISPRz database $^{60}$ https://research.nhgri.nih.gov/CRISPRz/). CRISPR mutation rates for founder embryos were analyzed by calculating the percentage of mutant signal over the total signal ${ }^{61}$.

\section{Biological materials and the zebrafish transgenic lines}

The biological dyes used in this study were: Yopro-1 (Life Technologies. Cat\#: Y3603), ProLong Gold Antifade Mountant with DAPI (Vector Laboratories. Cat\#: H1200). Chemical compounds used in this study were all purchased from Sigma: copper sulfate (C1297), antimycin A (A8674), cycloheximide (C7698), AG1478 (T4182), DAPT (D5942), dexamethasone (D4902), prednisolone (P6004), 1-azakenpaullone (A3734), BIO (B1686), IWR1 (I0161), SU5402 (SML0443), SB505124 (S4696), $\mathrm{H}_{2} \mathrm{O}_{2}$ (216763), NAC (A7250), and GSH (G4251). All chemicals except NAC and GSH were dissolved in DMSO. NAC and GSH were dissolved in embryo media 1x Holtfreter's buffer. Chemical treatments were carried out in embryo media, with the doses and durations listed in Supplementary Table 3. Mock treatments were carried out by adding an equal amount of the corresponding solvents. The zebrafish

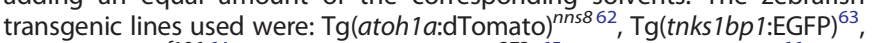
$\mathrm{Tg}(\mathrm{clndb} \text { :GFP })^{\mathrm{zf1} 106} 64$ Tg(pou4f3:GAP-GFP) $)^{\mathrm{s} 273 \mathrm{t} 65}$. Tg(SqET20:EGFP) $)^{66}$, Tg (foxd3:GFP) ${ }^{\text {zf1567 }}, \mathrm{Tg}\left(\right.$ fabp 10a:CFP-NTR) ${ }^{593168}$. Imaging of the transgenic embryos was conducted using either an inverted Zeiss Axiophot or a Zeiss LSM 880 confocal microscope.

\section{Hair cell and neuromast quantification}

Hair cell staining and quantification were as described ${ }^{69}$. Briefly, for analyzing hair cell development, embryos from heterozygotic incrosses were cultured until $5 \mathrm{dpf}$, and then placed in a cell strainer (BD Falcon) for staining with $2 \mu \mathrm{M}$ YoPro-1 for $30 \mathrm{~min}$. Lateral line neuromasts P1, P2, P4, and P5 in each embryo were used for hair cell counting using an inverted Zeiss Axiophot. The number of neuromasts in the lateral line of each embryo was also counted for studying neuromast formation. For hair cell regeneration analysis, embryos from heterozygotic incrosses at $5 \mathrm{dpf}$ were treated with the ototoxin copper sulfate at $10 \mu \mathrm{M}$ for $2 \mathrm{~h}$ except when otherwise indicated, recovered for $48 \mathrm{~h}$, and then counted for the regenerated hair cells in the lateral line neuromasts P1, P2, P4, and P5. Approximately 40 embryos were used for each of the analyses except when otherwise indicated. The average number of hair cells and the standard error of the mean (s.e.m.) are shown in the graphs.

\section{Immunohistochemical staining}

Hair cell staining for fixed zebrafish tissues was performed with a combination of antibodies against hair cell soma-1 (Developmental Studies Hybridoma Bank. Cat\#: HCS-1, $1 \mu \mathrm{g} / \mathrm{ml}$ ) and myosin-Vlla (Developmental Studies Hybridoma Bank. Cat\#: 138-1, $1 \mu \mathrm{g} / \mathrm{ml}$ ), followed by an Alexa 488-labeled

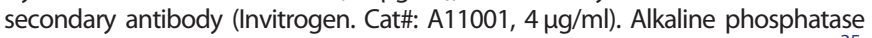
staining for lateral line neuromasts was performed as previously reported ${ }^{35}$. Lateral line axons were stained with an antibody against acetylated tubulin (Sigma. Cat\#: T7451, 1:1000 dilution) and a secondary antibody conjugated with Alexa 594 (Invitrogen. Cat\#: A11012, 4 $\mathrm{g} / \mathrm{ml}$ ). Proliferating cells were labeled with the Click-It EdU Alexa Fluor 594 Imaging Kit (Life Science. Cat\#: C10339), following the manufacturer's instructions. Embryos were prepared by exposure to $500 \mu \mathrm{M}$ of EdU in 1x Holtfreter's buffer with 15\% DMSO in an ice bath for 30 minutes, recovered for $3 \mathrm{~h}$, and then fixed in $4 \%$ paraformaldehyde overnight. The stained embryos were mounted with ProLong Gold Antifade Mountant with DAPI on a microscope slide and imaged with a Zeiss LSM 880 confocal microscope. The neuromast images were then used for EdU-positive cell quantification. For the $s m n 1$ mutation, neuromasts were additionally labeled by $\mathrm{Tg}(\mathrm{cldnb}$ :GFP). For the gemin5 mutation, neuromasts were additionally labeled by $\mathrm{Tg}($ tnks1bp1:EGFP).

\section{Quantifying development and regeneration of caudal fin}

Caudal fin development and regeneration were analyzed as previously described $^{14}$. In brief, embryos were obtained from a pair of heterozygous parents. Fin development was measured at $5 \mathrm{dpf}$, using the posterior of pigment gap as a positional reference. For the regeneration analysis, amputation was performed at $3 \mathrm{dpf}$, at the posterior end of ventral pigment break. The regeneration was measured at $7 \mathrm{dpf}$, continuing to use the anterior end of pigment gap as a positional reference. ImageJ was used for quantifying the fin areas. All analyzed embryos were genotyped. Graph shows the mean and s.e.m., based on the quantification data from $\sim 10$ embryos per genotype.

\section{Quantification of development and regeneration of liver}

Liver development and regeneration were tested using the transgene $\mathrm{Tg}$ (fabp 10a:CFP-NTR) ${ }^{68}$. The embryos used for the analysis were the CFPpositive embryos obtained from a pair of parents with one carrying the heterozygous gene mutation and the other carrying both the heterozygous mutation and an allele of Tg(fabp10a:CFP-NTR). Liver size was measured at 5 dpf. For liver regeneration analysis, the embryos were treated with $10 \mathrm{mM}$ metronidazole for 1.5 days at $3 \mathrm{dpf}$ and analyzed for regeneration at $7 \mathrm{dpf}$. All analyzed embryos were imaged at a lateral view with head facing right under a Zeiss Axiophot fluorescent microscope, and afterwards genotyped. ImageJ was used to measure the liver areas. Approximately 45 CFP-positive embryos were used for each analysis. Graph shows the mean and s.e.m.

\section{RNA-Seq analysis}

The embryos used for RNA-Seq were produced from a cross of a single pair of heterozygous parents, exposed to $10 \mu \mathrm{M}$ copper sulfate for $2 \mathrm{~h}$ at $5 \mathrm{dpf}$, and then subjected to caudal fin biopsy for genotyping and the body stored in Qiazol (Qiagen. Cat\#: 79306) at 7 dpf. Afterwards the wild-type and homozygous mutant embryos were pooled together and used for total RNA extraction by using Qiagen miRNeasy Mini Kit (Cat\#: 217004). The total RNA with an integrity score (RIN) over 9 were used for RNA-Seq analysis. For the RNA-Seq data analysis, reads were mapped to the reference genome (GRCz10, Ensembl release 91, without pseudogenes) using STAR v2.6.1c with the default settings. Expression abundance was estimated using RSEM. Differentially expressed genes were identified using $R$ package "DESeq2" for either the wildtype-mutant pair or all the samples combined. We defined a gene S1-up (or G3-up, G5-up) when it was significantly ( $\mathrm{fdr}<0.05$ ) up-regulated in the regeneration gene mutants $\mathrm{S} 1$ (or G3, G5) and not significantly ( $p \geq 0.1$ and $\mathrm{fdr} \geq 0.25$ ) in any of the nonregeneration gene mutants and the log2-fold change is not greater than log2(fold change of S1) - 0.2 (or G3,G5). We defined a gene S1-down when it was significantly down-regulated in the regeneration gene mutants $\mathrm{S} 1$ (or G3, G5) and not in any of the non-regeneration gene mutants and the log2-fold change is not greater than log2(fold change of $\mathrm{S} 1$ ) +0.2 (or G3, $\mathrm{G5}$ ). We labeled these $\mathrm{S} 1 / \mathrm{G} 3 / \mathrm{G} 5$-up/down genes as the regenerationassociated up/down genes. The regeneration-associated genes were then used for KEGG pathway, gene ontology, and InterPro analysis (Fisher's exact test FDR < 0.05). Gene set enrichment analysis was implemented for each wildtype-mutant pair. Gene sets with odds ratio $>1$ and (FDR $>0.1$ or 
$P$-value $>0.01$ ) in any of $\mathrm{S} 1, \mathrm{G} 3, \mathrm{G} 5$ wildtype-mutant pair but less significant in all of G2, G4, G6, G7 pairs were labeled as the enriched gene sets. Gene sets with fewer than 10 or more than 500 genes or with the regenerationassociated genes fewer than 3 were removed.

\section{Statistical analyses}

A Student $t$-test (two tailed) was used for comparison between two samples. One-way ANOVA was used when comparing multiple samples. A difference was considered significant when $P$ value was $<0.05$. Dotted box plot graphs were made using R. Center lines indicate the median. Asterisks and short lines were used to indicate a significant difference between two groups. ns, $P \geq 0.05 ;{ }^{*} P<0.05 ;{ }^{* *} P<0.01 ;{ }^{* * *} P<0.001 ; * * * * P<0.0001$. Each experiment presented was repeated at least twice, with the replicates showing statistical significance each time.

\section{Reporting summary}

Further information on research design is available in the Nature Research Reporting Summary linked to this article.

\section{DATA AVAILABILITY}

All RNA-seq data deposited to GEO under access number: GSE134187. Sequence and mutagenic activity of all sgRNA CRISPR guides used in this study available at the CRISPRz database https://research.nhgri.nih.gov/CRISPRz/.

Received: 20 August 2019; Accepted: 27 January 2020; Published online: 25 March 2020

\section{REFERENCES}

1. Simone, C. et al. Is spinal muscular atrophy a disease of the motor neurons only: pathogenesis and therapeutic implications? Cell. Mol. Life Sci. 73, 1003-1020 (2016).

2. Van Alstyne, M. \& Pellizzoni, L. Advances in modeling and treating spinal muscular atrophy. Curr. Opin. Neurol. 29, 549-556 (2016).

3. Li, D. K., Tisdale, S., Lotti, F. \& Pellizzoni, L. SMN control of RNP assembly: from post-transcriptional gene regulation to motor neuron disease. Semin. Cell Dev. Biol. 32, 22-29 (2014).

4. Yong, J., Kasim, M., Bachorik, J. L., Wan, L. \& Dreyfuss, G. Gemin5 delivers snRNA precursors to the SMN complex for snRNP biogenesis. Mol. Cell 38, 551-562 (2010).

5. Bernabo, P. et al. In vivo translatome profiling in spinal muscular atrophy reveals a role for SMN protein in ribosome biology. Cell Rep. 21, 953-965 (2017)

6. Chaytow, H., Huang, Y. T., Gillingwater, T. H. \& Faller, K. M. E. The role of survival motor neuron protein (SMN) in protein homeostasis. Cell. Mol. Life Sci. 75, 3877-3894 (2018).

7. Fallini, C., Bassell, G. J. \& Rossoll, W. Spinal muscular atrophy: the role of SMN in axonal mRNA regulation. Brain Res. 1462, 81-92 (2012).

8. Setola, V. et al. Axonal-SMN (a-SMN), a protein isoform of the survival motor neuron gene, is specifically involved in axonogenesis. Proc. Natl Acad. Sci. USA 104, 1959-1964 (2007).

9. Curmi, F. \& Cauchi, R. J. The multiple lives of DEAD-box RNA helicase DP103/ DDX20/Gemin3. Biochem. Soc. Trans. 46, 329-341 (2018).

10. Francisco-Velilla, R., Azman, E. B. \& Martinez-Salas, E. Impact of RNA-protein interaction modes on translation control: the versatile multidomain protein Gemin5. Bioessays 41, e1800241 (2019).

11. Garcia-Moreno, M. et al. System-wide profiling of RNA-binding proteins uncovers key regulators of virus infection. Mol. Cell 74, 196-211 e111 (2019).

12. Pineiro, D., Fernandez-Chamorro, J., Francisco-Velilla, R. \& Martinez-Salas, E. Gemin5: a multitasking RNA-binding protein involved in translation control. Biomolecules 5, 528-544 (2015).

13. Zhang, Z. et al. SMN deficiency causes tissue-specific perturbations in the repertoire of snRNAs and widespread defects in splicing. Cell 133, 585-600 (2008).

14. Pei, W. et al. Guided genetic screen to identify genes essential in the regeneration of hair cells and other tissues. NPJ Regen. Med. 3, 11 (2018).

15. Yang, C. H., Schrepfer, T. \& Schacht, J. Age-related hearing impairment and the triad of acquired hearing loss. Front. Cell. Neurosci. 9, 276 (2015).

16. Kniss, J. S., Jiang, L. \& Piotrowski, T. Insights into sensory hair cell regeneration from the zebrafish lateral line. Curr. Opin. Genet. Dev. 40, 32-40 (2016).

17. Lush, M. E. et al. scRNA-Seq reveals distinct stem cell populations that drive hair cell regeneration after loss of Fgf and Notch signaling. Elife 8, https://doi.org/ 10.7554/eLife.44431 (2019).
18. Smith, M. E., Groves, A. K. \& Coffin, A. B. Editorial: sensory hair cell death and regeneration. Front. Cell. Neurosci. 10, 208 (2016).

19. Behra, M. et al. Phoenix is required for mechanosensory hair cell regeneration in the zebrafish lateral line. PLoS Genet. 5, e1000455 (2009).

20. Pei, W. et al. Extracellular HSP60 triggers tissue regeneration and wound healing by regulating inflammation and cell proliferation. NPJ Regen. Med. 1, https://doi. org/10.1038/npjregenmed.2016.13 (2016).

21. Chen, C. H. \& Poss, K. D. Regeneration genetics. Annu. Rev. Genet. 51, 63-82 (2017).

22. Mokalled, M. H. \& Poss, K. D. A regeneration toolkit. Dev. Cell 47, 267-280 (2018).

23. Borg, R. M., Bordonne, R., Vassallo, N. \& Cauchi, R. J. Genetic interactions between the members of the SMN-gemins complex in Drosophila. PLOS ONE 10, e0130974 (2015).

24. O'Hern, P. J. et al. Decreased microRNA levels lead to deleterious increases in neuronal M2 muscarinic receptors in Spinal Muscular Atrophy models. Elife 6 https://doi.org/10.7554/eLife.20752 (2017)

25. Workman, E., Kalda, C., Patel, A. \& Battle, D. J. Gemin5 binds to the survival motor neuron mRNA to regulate SMN expression. J. Biol. Chem. 290, 15662-15669 (2015).

26. Chari, A. et al. An assembly chaperone collaborates with the SMN complex to generate spliceosomal SnRNPs. Cell 135, 497-509 (2008).

27. Pellizzoni, L., Yong, J. \& Dreyfuss, G. Essential role for the SMN complex in the specificity of snRNP assembly. Science 298, 1775-1779 (2002).

28. Whitfield, T. T. et al. Mutations affecting development of the zebrafish inner ear and lateral line. Development 123, 241-254 (1996).

29. Rojas-Munoz, A. et al. ErbB2 and ErbB3 regulate amputation-induced proliferation and migration during vertebrate regeneration. Dev. Biol. 327, 177-190 (2009).

30. Chua, J. S., Liew, H. P., Guo, L. \& Lane, D. P. Tumor-specific signaling to p53 is mimicked by $\mathrm{Mdm} 2$ inactivation in zebrafish: insights from $\mathrm{mdm} 2$ and $\mathrm{mdm} 4$ mutant zebrafish. Oncogene 34, 5933-5941 (2015).

31. Van Alstyne, M. et al. Dysregulation of Mdm2 and Mdm4 alternative splicing underlies motor neuron death in spinal muscular atrophy. Genes Dev. 32, 1045-1059 (2018).

32. Young, P. J. et al. A direct interaction between the survival motor neuron protein and p53 and its relationship to spinal muscular atrophy. J. Biol. Chem. 277, 2852-2859 (2002).

33. Berghmans, S. et al. tp53 mutant zebrafish develop malignant peripheral nerve sheath tumors. Proc. Natl Acad. Sci. USA 102, 407-412 (2005).

34. Ramanagoudr-Bhojappa, R. et al. Multiplexed CRISPR/Cas9-mediated knockout of 19 Fanconi anemia pathway genes in zebrafish revealed their roles in growth sexual development and fertility. PLoS Genet. 14, e1007821 (2018).

35. Lush, M. E. \& Piotrowski, T. ErbB expressing Schwann cells control lateral line progenitor cells via non-cell-autonomous regulation of Wnt/beta-catenin. Elife 3, e01832 (2014).

36. Lopez-Schier, H. \& Hudspeth, A. J. Supernumerary neuromasts in the posterior lateral line of zebrafish lacking peripheral glia. Proc. Natl Acad. Sci. USA 102 1496-1501 (2005).

37. Perlin, J. R., Lush, M. E., Stephens, W. Z., Piotrowski, T. \& Talbot, W. S. Neuronal Neuregulin 1 type III directs Schwann cell migration. Development 138 4639-4648 (2011).

38. Grant, K. A., Raible, D. W. \& Piotrowski, T. Regulation of latent sensory hair cell precursors by glia in the zebrafish lateral line. Neuron 45, 69-80 (2005).

39. Han, Y. et al. Vitamin D stimulates cardiomyocyte proliferation and controls organ size and regeneration in zebrafish. Dev. Cell https://doi.org/10.1016/j. devcel.2019.01.001 (2019).

40. Burghes, A. H. \& Beattie, C. E. Spinal muscular atrophy: why do low levels of survival motor neuron protein make motor neurons sick? Nat. Rev. Neurosci. 10, 597-609 (2009)

41. Jablonka, S. et al. Gene targeting of Gemin2 in mice reveals a correlation between defects in the biogenesis of $U$ snRNPs and motoneuron cell death. Proc. Natl Acad. Sci. USA 99, 10126-10131 (2002).

42. Mouillet, J. F. et al. DEAD-box protein-103 (DP103, Ddx20) is essential for early embryonic development and modulates ovarian morphology and function. Endocrinology 149, 2168-2175 (2008).

43. Liu, Q. \& Dreyfuss, G. A novel nuclear structure containing the survival of motor neurons protein. EMBO J. 15, 3555-3565 (1996).

44. McWhorter, M. L., Boon, K. L., Horan, E. S., Burghes, A. H. \& Beattie, C. E. The SMN binding protein Gemin2 is not involved in motor axon outgrowth. Dev. Neurobiol. 68, 182-194 (2008).

45. McWhorter, M. L., Monani, U. R., Burghes, A. H. \& Beattie, C. E. Knockdown of the survival motor neuron ( $\mathrm{Smn}$ ) protein in zebrafish causes defects in motor axon outgrowth and pathfinding. J. Cell Biol. 162, 919-931 (2003).

46. Carrel, T. L. et al. Survival motor neuron function in motor axons is independent of functions required for small nuclear ribonucleoprotein biogenesis. J. Neurosci. 26, 11014-11022 (2006). 
47. Francisco-Velilla, R., Fernandez-Chamorro, J., Dotu, I. \& Martinez-Salas, E. The landscape of the non-canonical RNA-binding site of Gemin5 unveils a feedback loop counteracting the negative effect on translation. Nucleic Acids Res. 46, 7339-7353 (2018).

48. Francisco-Velilla, R., Fernandez-Chamorro, J., Ramajo, J. \& Martinez-Salas, E. The RNA-binding protein Gemin5 binds directly to the ribosome and regulates global translation. Nucleic Acids Res. 44, 8335-8351 (2016).

49. Mei, L. \& Nave, K. A. Neuregulin-ERBB signaling in the nervous system and neuropsychiatric diseases. Neuron 83, 27-49 (2014).

50. Cai, Q. et al. Epstein-Barr virus nuclear antigen 3 C stabilizes Gemin3 to block p53mediated apoptosis. PLoS Pathog. 7, e1002418 (2011).

51. Battle, D. J., Kasim, M., Wang, J. \& Dreyfuss, G. SMN-independent subunits of the SMN complex. Identification of a small nuclear ribonucleoprotein assembly intermediate. J. Biol. Chem. 282, 27953-27959 (2007).

52. Kroiss, M. et al. Evolution of an RNP assembly system: a minimal SMN complex facilitates formation of UsnRNPs in Drosophila melanogaster. Proc. Natl Acad. Sci. USA 105, 10045-10050 (2008).

53. Maslon, M. M., Heras, S. R., Bellora, N., Eyras, E. \& Caceres, J. F. The translational landscape of the splicing factor SRSF1 and its role in mitosis. Elife e02028 (2014).

54. Palangat, M. et al. The splicing factor U2AF1 contributes to cancer progression through a noncanonical role in translation regulation. Genes Dev. 33, 482-497 (2019).

55. Sanford, J. R., Gray, N. K., Beckmann, K. \& Caceres, J. F. A novel role for shuttling SR proteins in mRNA translation. Genes Dev. 18, 755-768 (2004).

56. Wishart, T. M. et al. Dysregulation of ubiquitin homeostasis and beta-catenin signaling promote spinal muscular atrophy. J. Clin. Invest. 124, 1821-1834 (2014).

57. Ramser, J. et al. Rare missense and synonymous variants in UBE1 are associated with X-linked infantile spinal muscular atrophy. Am. J. Hum. Genet. 82, 188-193 (2008).

58. Kimmel, C. B., Ballard, W. W., Kimmel, S. R., Ullmann, B. \& Schilling, T. F. Stages of embryonic development of the zebrafish. Dev. Dyn. 203, 253-310 (1995).

59. Varshney, G. K. et al. A high-throughput functional genomics workflow based on CRISPR/Cas9-mediated targeted mutagenesis in zebrafish. Nat. Protoc. 11, 2357-2375 (2016)

60. Varshney, G. K. et al. CRISPRz: a database of zebrafish validated sgRNAs. Nucleic Acids Res. 44, D822-826 (2016).

61. Carrington, B., Varshney, G. K., Burgess, S. M. \& Sood, R. CRISPR-STAT: an easy and reliable PCR-based method to evaluate target-specific sgRNA activity. Nucleic Acids Res. 43, e157 (2015).

62. Kani, S. et al. Proneural gene-linked neurogenesis in zebrafish cerebellum. Dev. Biol. 343, 1-17 (2010).

63. Behra, M. et al. Transcriptional signature of accessory cells in the lateral line, using the Tnk1bp1:EGFP transgenic zebrafish line. BMC Dev. Biol. 12, 6 (2012).

64. Haas, P. \& Gilmour, D. Chemokine signaling mediates self-organizing tissue migration in the zebrafish lateral line. Dev. Cell 10, 673-680 (2006).

65. Xiao, T., Roeser, T., Staub, W. \& Baier, H. A GFP-based genetic screen reveals mutations that disrupt the architecture of the zebrafish retinotectal projection. Development 132, 2955-2967 (2005).

66. Parinov, S., Kondrichin, I., Korzh, V. \& Emelyanov, A. Tol2 transposon-mediated enhancer trap to identify developmentally regulated zebrafish genes in vivo. Dev. Dyn. 231, 449-459 (2004).

67. Gilmour, D. T., Maischein, H. M. \& Nusslein-Volhard, C. Migration and function of a glial subtype in the vertebrate peripheral nervous system. Neuron 34, 577-588 (2002).

68. Choi, T. Y., Khaliq, M., Ko, S., So, J. \& Shin, D. Hepatocyte-specific ablation in zebrafish to study biliary-driven liver regeneration. J. Vis. Exp. e52785, https://doi. org/10.3791/52785 (2015).

69. Pei, W. et al. Additive reductions in zebrafish PRPS1 activity result in a spectrum of deficiencies modeling several human PRPS1-associated diseases. Sci. Rep. 6, 29946 (2016).

\section{ACKNOWLEDGEMENTS}

We would like to thank MaryPat Jones, Blake Carrington, Kevin Bishop and Raman Sood from the NHGRI Zebrafish Core for mutation genotyping; Suiyuan Zhang from the NHGRI Bioinformatics Core for CRISPRz database management; Alisha Beirl and Katie Kindt from the NIDCD for mechanistic studies; Daniel Green, Justin Frye, Jason Frye, Hillary Mahon, Paulina Capar, and Justin Glover in Charles River for fish care; and the members of Burgess laboratory for helpful discussion. We have complied with all relevant ethical regulations regarding animal use and all animal experiments were approved by the National Human Genome Research Institute's Animal Care and Use Committee (protocol \#G-01-3). This research was supported by the Intramural Research Program of the National Human Genome Research Institute (ZIAHG200386-06).

\section{AUTHOR CONTRIBUTIONS}

W.P. and S.M.B. designed the study. W.P., L.X., C.C.S., and K.P.P. performed zebrafish experiments. Z.C. conducted bioinformatics analysis. S.W. assisted with confocal imaging. NISC Comparative Sequencing Program performed RNA-Seq. W.P. and S.M. B. prepared the manuscript. All authors approved the submission of the manuscript.

\section{COMPETING INTERESTS}

The authors declare no competing interests.

\section{ADDITIONAL INFORMATION}

Supplementary information is available for this paper at https://doi.org/10.1038/ s41536-020-0089-0.

Correspondence and requests for materials should be addressed to S.M.B.

Reprints and permission information is available at http://www.nature.com/ reprints

Publisher's note Springer Nature remains neutral with regard to jurisdictional claims in published maps and institutional affiliations.

(i) Open Access This article is licensed under a Creative Commons Attribution 4.0 International License, which permits use, sharing, adaptation, distribution and reproduction in any medium or format, as long as you give appropriate credit to the original author(s) and the source, provide a link to the Creative Commons license, and indicate if changes were made. The images or other third party material in this article are included in the article's Creative Commons license, unless indicated otherwise in a credit line to the material. If material is not included in the article's Creative Commons license and your intended use is not permitted by statutory regulation or exceeds the permitted use, you will need to obtain permission directly from the copyright holder. To view a copy of this license, visit http://creativecommons. org/licenses/by/4.0/

This is a U.S. government work and not under copyright protection in the U.S.; foreign copyright protection may apply 2020

\section{NISC COMPARATIVE SEQUENCING PROGRAM}

Beatrice B. Barnabas ${ }^{3}$, Sean Black ${ }^{3}$, Gerard G. Bouffard ${ }^{3}$, Shelise Y. Brooks ${ }^{3}$, Holly Coleman ${ }^{3}$, Lyudmila Dekhtyar ${ }^{3}$, Xiaobin Guan $^{3}$, Joel $\mathrm{Han}^{3}$, Shi-ling $\mathrm{Ho}^{3}$, Richelle Legaspi ${ }^{3}$, Quino L. Maduro ${ }^{3}$, Catherine A. Masiello ${ }^{3}$, Jennifer C. McDowell ${ }^{3}$, Casandra Montemayor ${ }^{3}$, James C. Mullikin ${ }^{3}$, Morgan Park ${ }^{3}$, Nancy L. Riebow ${ }^{3}$, Karen Schandler ${ }^{3}$, Chanthra Scharer ${ }^{3}$, Brian Schmidt ${ }^{3}$, Christina Sison $^{3}$, Sirintorn Stantripop ${ }^{3}$, James W. Thomas ${ }^{3}$, Pamela J. Thomas ${ }^{3}$, Meghana Vemulapalli ${ }^{3}$ and Alice C. Young ${ }^{3}$

${ }^{3} \mathrm{NIH}$ Intramural Sequencing Center, National Institutes of Health, Bethesda, USA 